Z. Phys. D - Atoms, Molecules and Clusters 2, 257-274 (1986)

Atoms, Molecules

\title{
Experimental Characterization of the Xe $5 p$ Photoionization by Angle- and Spin-Resolved Photoelectron Spectroscopy *
}

\author{
Ch. Heckenkamp ${ }^{1}$, F.Schäfers ${ }^{2}$, G. Schönhense, and U. Heinzmann \\ Fakultät für Physik der Universität Bielefeld, Federal Republic of Germany \\ Fritz-Haber-Institut der MPG, Berlin, Federal Republic of Germany
}

Received March 12, 1986

Spin- and angle-resolved photoelectron spectroscopy with elliptically polarized radiation has been used to fully characterize the dynamics of photoelectron emission from free $\mathrm{Xe}$ atoms in the $5 p$-autoionization and continuum region. An advantageous reaction geometry and its experimental realization at the storage ring BESSY are discussed. The three independent experimental parameters which characterize the angular dependence of the photoelectron spin-polarization vector are reported for the wavelength range from $100 \mathrm{~nm}$ to $40 \mathrm{~nm}$. The results are compared with theoretical predictions based on RRPA-, RPAE- and semiempirical MQDT-calculations. The combination of existing data for the differential photoionization cross section with the spinpolarization parameters is used to completely decouple the photoionization channels: The transition matrix elements and their relative phases are determined separately for every single dissociation channel. The results are discussed in the context of the MQDT. Correlation effects and the influence of spin-orbit interaction on the continuum states most clearly show up when the Dill-Fano angular-momentum-transfer formalism is applied.

PACS: $3280 \mathrm{~F} ; 3280 \mathrm{D}$

\section{Introduction}

The interaction of radiation with matter plays an important role in many physical, chemical or technological problems of practical importance. The investigation of the photoionization of free atoms is of fundamental interest in this context. In addition, it gives detailed insight into the electronic structure of atoms [1].

Besides the conventional absorption experiments, the detection of reaction products (photoelectrons, photoions, fluorescence photons) is of growing im-

* This paper is dedicated to Professor Dr. J. Geiger, University of Kaiserslautern, Germany, who died December 20th 1985. His expertise of the Multichannel Quantum Defect Theory has guided the authors since 1977 and has strongly influenced the quantummechanical treatment of the photoionization of xenon

Present adress:

${ }^{1}$ Gesellschaft für Automation und Organisation, Euckenstrasse 12, D-8000 München 70, FRG

${ }^{2}$ BESSY, Lentzeallee 100, D-1000 Berlin 33, FRG portance in this field [2]. An important and well established technique of this kind is angle resolved photoelectron spectroscopy, where the photoemission process is studied by observation of the kinetic energy and of the emission angle of the photoelectron, i.e. of its momentum. A further observable of the photoelectron is its spin orientation. Spin-resolved electron detection will usually lead to information independent of that obtained by photoelectron-intensity measurements.

The subject of the present paper is an experiment at xenon atoms, where angle-resolved photoelectron spectroscopy, spin-resolved electron detection and the use of synchrotron radiation have been combined. Synchrotron radiation is needed for this kind of experiments, since the full information contained in the spin-orientation of photoelectrons can only be exploited when circularly polarized radiation is used for ionization. This requirement is exclusively met by synchrotron radiation for photon energies above $10 \mathrm{eV}$. In addition, synchrotron radiation provides a 
tuneable light source over a wide wavelength range in the vacuum ultraviolet. This becomes important especially when the energy dependence of the photoionization process is essential, for example in resonance regions.

First experimental investigations of spin-polarized photoelectrons from free atoms began about 1970 [3]. The existence of polarized photoelectrons in $s$-shell photoionization of alkali atoms by circularly polarized light was theoretically predicted by Fano $[4,5]$ and experimentally verified for cesium $[6,7]$. In these and the following investigations of the so-called "Fano-effect" [8,9] the angle-integrated mean value of the spin-polarization was measured, which is proportional to the degree of circular polarization of the ionizing radiation. The Fanoeffect is therefore sometimes interpreted as a spinpolarization transfer from the ionizing light to the photoelectrons, the transfer mechanism being the spin-orbit interaction. In a first experiment at lead [10] it was shown that photoelectrons, even when produced by unpolarized light, can be spin-polarized, if the emission is studied angle-resolved. Spinorbit interaction again is the reason for the spinpolarization, which is caused by interference between different continuum wavefunctions. Since then experiments have been performed at various targets, where the spin-polarization transfer with circularly polarized radiation [11-22], as well as the photoelectron spin-polarization with unpolarized and linearly polarized light $[17,18,21,23-27]$ have been investigated.

Theoretical treatments of spin-polarized photoelectron emission from unpolarized atomic targets have been performed by Stuart [28], Brehm [29], Jacobs [30], Cherepkov [31, 32], Lee [33], and Klar [34] in dipole approximation and in non-relativistic formulation. Huang $[35,36]$ treats the problem using a relativistic approach for higher multipole fields and derives, as later also Cherepkov [37], relations for any state of polarization of the ionizing radiation. Based on this work, several numerical calculations of the spin-polarization of photoelectrons as function of photon energy have been performed for different atomic systems [32, 37-47]. There also exist calculations for autoionization resonances [33, 48-53, 64].

The angular dependences of the three components of the photoelectron spin-polarization vector can be characterized by functions containing Legendre-polynomials and three energy dependent spin-parameters $[9,26,54]$, which appear together only in angle-resolved measurements with circularly polarized radiation. In this paper we report on the experimental investigation of the wavelength depen- dence of the three dynamical spin-parameters for photoionization of free xenon atoms in the outermost $5 p^{6}$ shell. The experimental results have already partly been published $[55,56]$. We combine these results with existing experimental data for the partial cross section $Q$ and the angular distribution of the photoelectrons, characterized by the asymmetry parameter $\beta$. From this data set, all transition matrix elements and phase-shift differences, which appear in the theoretical description of the $5 p$ photoionization of xenon can be determined separately $[33,39,57,58]$, in the autoionization range as well as in the open continuum. We discuss the results of this complete analysis in the framework of the quantum defect theory and compare them with theoretical calculations. Xenon is a well-suited target, since its partial cross section and asymmetry parameters are well known. The heavy xenon system should show significant relativistic and correlation effects, which strongly influence the dynamical spinparameters $[1,52,59,60]$ and can only correctly be treated by the highly sophisticated modern photoionization theories. The results should therefore be a sensitive test for the existing theories.

The intention of this paper is to explain the concept of spin- and angle-resolved photoelectron spectroscopy with circularly polarized synchrotron radiation and to show how and which experimental results can be used for the complete quantum mechanical characterization of the photoionization process. We also give a brief description of the experimental arrangement with emphasis on some critical details.

\section{Kinematics of the Photoionization Process}

The general relations for the dependence of the differential cross section and the three components of the spin-polarization vector of photoelectrons upon the reaction angle $\theta$, as given by Huang [36] for photoionization of unpolarized free atoms with elliptically polarized radiation of any degree of polarization, greatly simplify, when the reaction geometry shown in Fig. 1 is chosen. The reaction plane, spanned by the momentum vectors $\mathbf{k}_{i}$ of the incoming photon and $\mathbf{k}_{0}$ of the outgoing photoelectron, is rotated by $\pi / 4$ with respect to the major axis of the light-polarization ellipse. The reaction plane is the $x$ $-z$-plane of a right-handed laboratory coordinate system, where the $z$-axis coincides with the propagation direction of the photons and the origin is the interaction centre. The reaction angle $\theta$ is measured in the reaction plane from the $z$-axis to the momentum vector of the outgoing electron. The spin-polar- 
Fig. 1. Reaction geometry and spinpolarization vectors for photoionization with circularly polarized light. Photons are incident from the left

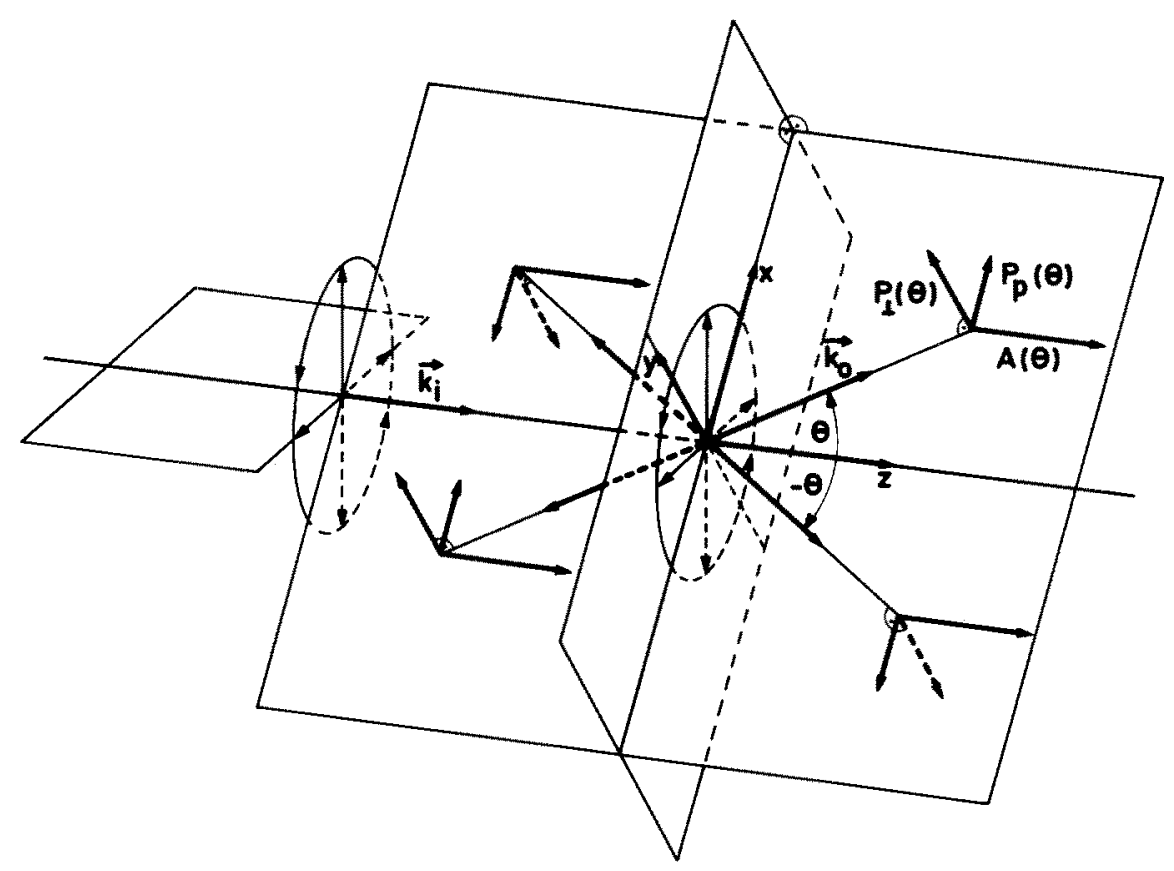

ization vector $\mathbf{P}(\theta)$ consists of three components $P_{\perp}(\theta)=P_{y}(\theta)$ perpendicular to the reaction plane, $A_{\mathrm{ell}}(\theta)=P_{z}(\theta)$ parallel to the photon momentum and $P_{p, \text { ell }}(\theta)=P_{x}(\theta)$ in the reaction plane, but perpendicular to the photon momentum. The general relations for the differential cross section and spin-polarization vector of the photoelectrons in this reaction geometry lead to:

$\frac{\mathrm{d} \sigma}{\mathrm{d} \Omega}(\theta)=\frac{Q}{4 \pi}\left(1-\frac{\beta}{2} P_{2}(\cos \theta)\right)=\frac{Q}{4 \pi} F(\theta, \beta)$

$P_{\perp}(\theta)=\frac{2 \xi \sin \theta \cos \theta}{F(\theta, \beta)}$

independent of helicity and degree of light polarization, and:

$A_{\text {ell }}(\theta)=\gamma\left|P_{\text {circ }}\right| \frac{A-\alpha P_{2}(\cos \theta)}{F(\theta, \beta)}-P_{\operatorname{lin}} \frac{2 \xi \sin ^{2} \theta}{F(\theta, \beta)}$

$P_{p, \text { ell }}(\theta)=\gamma\left|P_{\text {circ }}\right| \frac{-\frac{3}{2} \alpha \sin \theta \cos \theta}{F(\theta, \beta)}+P_{\text {lin }} \frac{2 \xi \sin \theta \cos \theta}{F(\theta, \beta)}$.

$Q$ is the integral cross-section, $\beta$ the asymmetry parameter of the differential cross-section and $P_{2}(\cos \theta)$ $=\frac{3}{2} \cos ^{2} \theta-\frac{1}{2}$ the second Legendre-polynomial. Equation (1) also holds for circularly polarized and unpolarized radiation, whereas for elliptically polarized light in general the degree of photon polarization and the azimuthal orientation of the reaction plane enter into the equations $[36,61-63,65-67$, 94]. In the particular reaction geometry chosen, however, these quantities neither appear in the differential cross-section nor in the denominator of the spin-polarization formulae. Even $P_{\perp}(\theta)$ is independent of the degree of photon polarization and is described by the spin-parameter $\xi$, which also appears in photoionization with linear [25] or unpolarized [23] light. Only when circularly polarized radiation is used, spin-polarization components in the reaction plane appear, the components $A_{\text {ell }}(\theta)$ and $P_{p \text {, ell }}(\theta)$ in Fig. 1. As additional quantities the parameters $A$ and $\alpha$ are used to parametrize the angular distributions of these spin-polarization components. $P_{\text {circ }}$ and $P_{\text {lin }}$ are the circular and linear degree of polarization; $\gamma$ is the helicity of the light: $\gamma$ $=+1$ for $\sigma^{+}$-light, that is photon spin and photon momentum are parallel, and $\gamma=-1$ for $\sigma^{-\prime}$-light. $A_{\text {ell }}(\theta)$ and $P_{p, \text { ell }}(\theta)$ contain a term proportional to $P_{\text {lin }}$ and $\xi$, as a consequence of the linearly polarized component in the elliptically polarized light. In contrast to the other terms, this term does not change sign, when $\sigma^{-}$-light is used instead of $\sigma^{+}$-light. As a consequence, the term proportional to $\gamma$ can be directly measured, basically by measuring with $\sigma^{+}$. light and $\sigma^{-}$-light and subtracting the results from each other. In this way, we get spin-polarization values which differ only by the factor $\left|P_{\text {circ }}\right|$ from those obtained with completely circularly polarized light, where Eqs. (1) and (2) remain valid, but $A_{\text {el1 }}(\theta)$ and $P_{p, \mathrm{ell}}(\theta)$ are substituted by $A(\theta)$ and $P_{p}(\theta)$ :

$A(\theta)=\gamma \frac{A-\alpha P_{2}(\cos \theta)}{1-\frac{\beta}{2} P_{2}(\cos \theta)}$ 
$P_{p}(\theta)=\gamma \frac{-\frac{3}{2} \alpha \sin \theta \cos \theta}{1-\frac{\beta}{2} P_{2}(\cos \theta)}$.

This case is depicted in Fig. 1 for the reaction angles $\theta, \pi-\theta,-\theta$ and $-(\pi-\theta)$ to show the symmetry properties of the spin-polarization components.

We note that the component $A(\theta)$ already contains the full information about the in-plane polarization components, since the parameter $\alpha$ characterizing $P_{p}(\theta)$ already appears in Eq. (5). The experimental verification of this relation is described in [55].

$A(\theta)$ is the only spin-polarization component which does not vanish when it is integrated over the full sphere:

$\int \mathrm{d} \Omega A(\theta)=\gamma A$.

This equation shows the possibility to determine $A$ in an experiment, where all photoelectrons are extracted by an electric field regardless of their direction of emission [7]. In an angle-resolving experiment, $A$ can be directly determined by measuring $A(\theta)$ at the so-called "magic angle" $\theta_{m}=54^{\circ} 44^{\prime}$, where $P_{2}\left(\cos \theta_{m}\right)$ vanishes:
$A\left(\theta_{m}\right)=\gamma A$.

In analogy, $\xi$ is determined from:

$P_{\perp}\left(\theta_{m}\right)=2 \xi \sin \theta_{m} \cos \theta_{m}=0.943 \xi$.

Further measurements of $A(\theta)$ at different angles allow the determination of the parameter $\alpha$.

Summarizing, angle-resolved measurements of the two spin-polarization components $P_{\perp}(\theta)$ and $A(\theta)$ for different emission angles $\theta$ yield the three dynamical spin-parameters $A, \alpha$ and $\xi$, which are needed for the complete quantum-mechanical characterization of the photoionization process.

\section{Experimental}

The reaction geometry described above has been realized at the dedicated storage ring BESSY in an experimental set-up, which is partly shown in Figs. 2 and 3. Monochromatized circularly polarized VUV synchrotron radiation, incident perpendicular to the drawing plane, crosses the atomic beam in the center of Fig. 2. The photoelectrons emitted in the reaction plane at an angle $\theta$ are energy analyzed in a simulated hemispherical electron spectrometer [68],

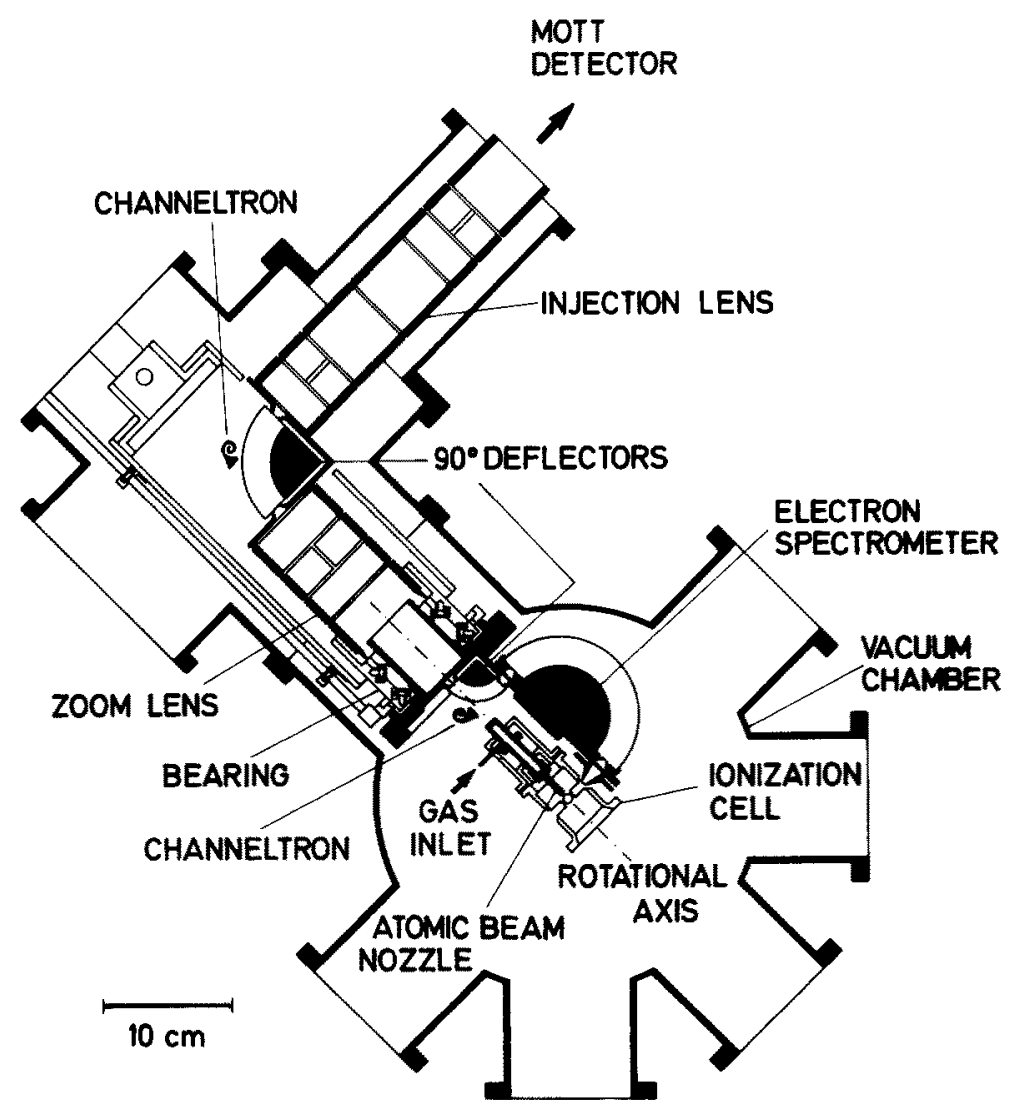

Fig. 2. Semi-technical drawing of the apparatus (without Mott detector). The photon momentum is normal to the drawing plane 
Fig. 3. Semi-technical drawing of the differential pumping system. The marginal rays correspond to the optical acceptance of the beamline

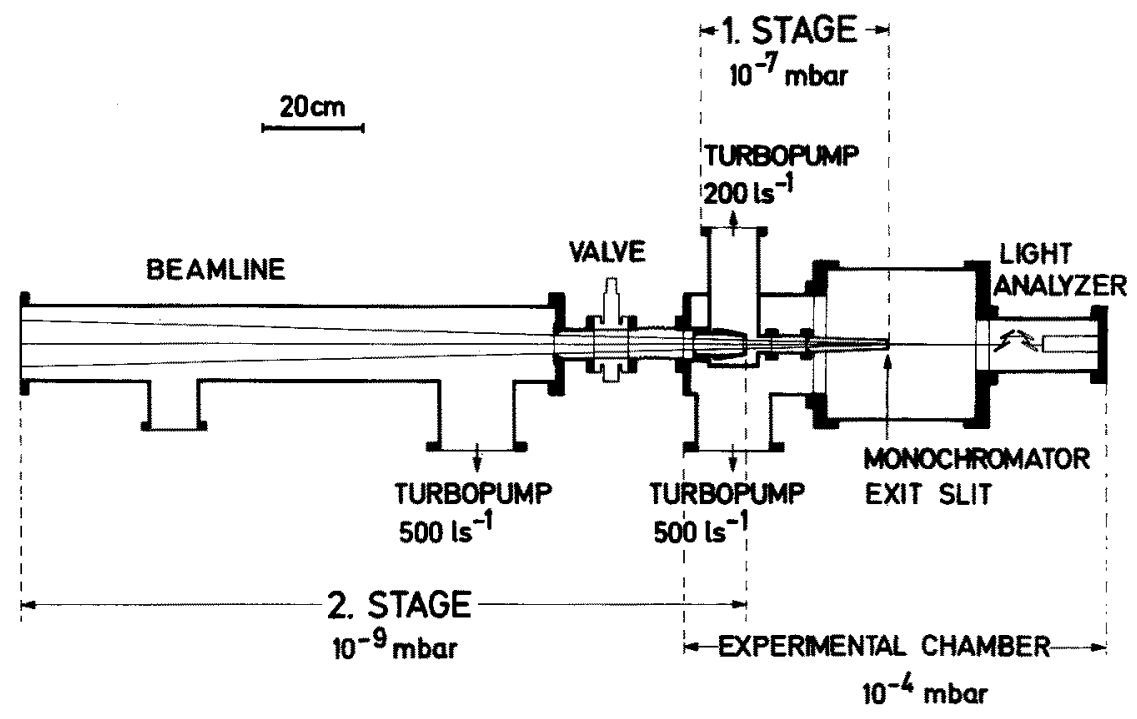

which is rotatable about the normal of the reaction plane as shown in Fig. 2. An electrostatic deflection by $90^{\circ}$ directs the electron beam along the axis of rotation of the electron spectrometer. After a second deflection by $90^{\circ}$ the electron beam is accelerated to $120 \mathrm{keV}$ and scattered at the gold foil of a Mott detector $[3,13]$, where the two transverse electron spin-polarization components $A(\theta)$ and $P_{\perp}(\theta)$ are simultaneously determined. A detailed description of the electron optical system, which allows angle-resolved measurements although light source and spinpolarization detector are fixed, is given in [69].

Since modern storage rings and monochromators are built to UHV-standards, a pressure of typically $10^{-4} \mathrm{mbar}$ in the gas-phase experiment must be pumped down to about $10^{-9} \mathrm{mbar}$ in the beamline. This is maintained by the differential pumping stage shown in Fig. 3, which has an optically free path corresponding to a divergence of the light beam of $60 \mathrm{mrad}$. The most important aperture in this double-stage system is the monochromator exit slit ( $2 \mathrm{~mm}$ $\times 5 \mathrm{~mm}$ ), which is integrated into the experimental chamber. The $6.5 \mathrm{~m}$ normal incidence monochromator $[70,71]$ with spherical mirror and plane grating $(1,200$ lines $/ \mathrm{mm})$, uses the electron beam in the storage ring as virtual entrance slit and forms a $1: 1$ image of the tangential point in the exit slit (MonkGillieson-mount). A bandpass of $0.5 \mathrm{~nm}$ in first and of $0.25 \mathrm{~nm}$ in second diffraction order has been measured. Apertures moveable in vertical direction in front of the monochromator are used to select radiation emitted above or below the storage ring plane, which has positive or negative helicity, respectively.

The degrees of circular and linear polarization of the light are routinely measured with a four-mirror

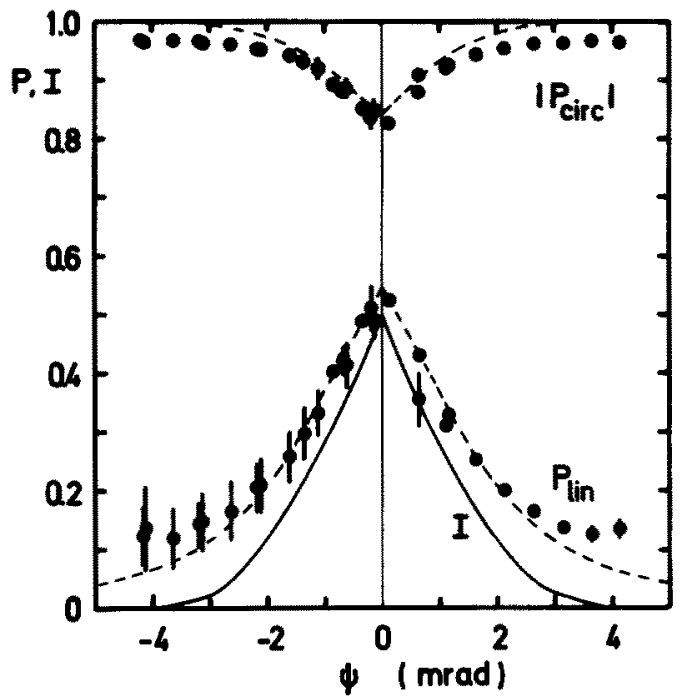

Fig. 4. Degree of circular and linear polarization for synchrotron radiation with $\lambda=100 \mathrm{~nm}$ from the storage ring BESSY (from [71]). Points, measurements with apertures accepting a vertical angular range from $\psi$ to $\pm 5 \mathrm{mrad}$ [55]; Dashed curve, calculated polarization of the radiation; Full curve, ratio of the corresponding measured photon intensity to the total intensity in the full vertical angular range from -5 to $+5 \mathrm{mrad}$

analyzer (see Fig. 3). As an example, Fig. 4 [71] shows the values measured in the vertical acceptance range from the angle $\psi$ above (for positive $\psi$ ) or below the storage-ring plane to $+5 \mathrm{mrad}$ or $-5 \mathrm{mrad}$, respectively. Figure 4 also shows the ratio $I$ of the intensity in this angular range to the total intensity in the full vertical acceptance of $\pm 5 \mathrm{mrad}$. Our measurements are usually performed with the apertures set to accept radiation above or below $\pm 1 \mathrm{mrad}$, which means that the degree of circular polarization is about $\pm 90 \%$, and about one third of 


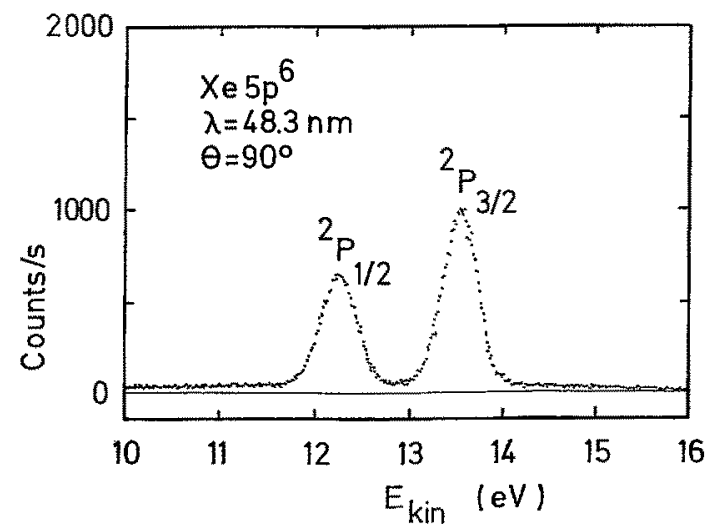

Fig. 5. Photoelectron spectrum for $\mathrm{Xe} 5 p$ in the continuum region excited with synchrotron radiation at $48.3 \mathrm{~nm}$. The electron emission angle $\theta$ is $90^{\circ}$ with respect to the photon momentum

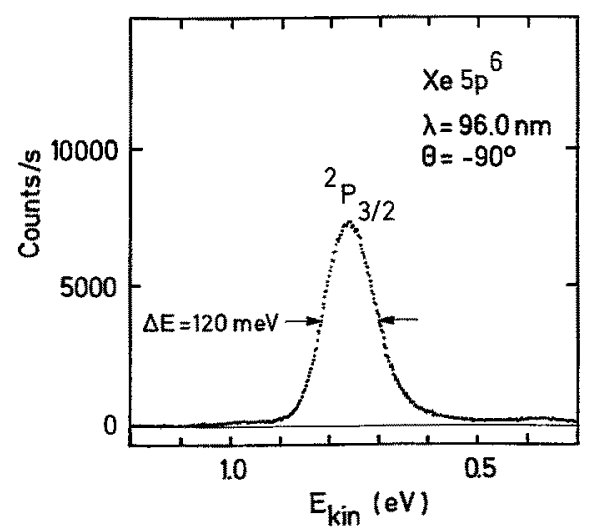

Fig. 6. Photoelectron spectrum for $\mathrm{Xe} 5 p$ in the autoionization region excited with synchrotron radiation at $96.0 \mathrm{~nm}$. The electron emission angle is $-90^{\circ}$ with respect to the photon momentum

the intensity emitted in the full vertical cone can be used. Further details on the mechanical and optical features of the monochromator are given elsewhere [71].

The photoionization measurements in the open continuum above the second ionization threshold of xenon could be performed with radiation of the first diffraction order, where photon fluxes on the order of $10^{11} \mathrm{~s}^{-1}$ were available with typical storage ring beam currents of $200 \mathrm{~mA}$. Second order radiation, however, had to be used to resolve the resonance structure in the autoionization range between the first and second ionization threshold. These measurements became possible when stored beam currents of $600 \mathrm{~mA}$ and more became routinely available, resulting in about $10^{11}$ photons per second of circularly polarized light behind the exit slit in second order.

Figures 5 and 6 show electron spectra for ionization of xenon in the $5 p$-shell in the continuous and in the autoionization range, respectively, recorded with a monitor channeltron behind the electron spectrometer (as shown in Fig. 2). The count rates of some $10^{3} \mathrm{~s}^{-1}$ are a factor of $10^{3}$ higher than those obtained in the Mott detector for the spin analysis. Typical count rates for the determination of the spin-polarizations ranged from $0.5 \mathrm{~s}^{-1}$ to $20 \mathrm{~s}^{-1}$ and were at least one order of magnitude higher than the corresponding background. In view of these values it is obvious that long counting times are required. This means that all components have to be reliable and must have good long-time stability. In particular, special care must be taken to design the monochromator and the electron optics for highest throughput, the atomic beam for high density, and the Mott detector for high efficiency. From this point of view, the experiment has certainly benefited much from the fact that monochromator and beamline could be tailored to the experiment and from the excellent running conditions of the storage ring BESSY.

\section{The Continuum Region}

\subsection{Experimental Results}

Photoionization of xenon atoms in the $5 p^{6}$-shell for photon energies above the second ionization threshold at $13.44 \mathrm{eV}$ is described by the reaction:

$\mathrm{Xe} 5 p^{6}\left({ }^{1} S_{0}\right)+h v \rightarrow \mathrm{Xe}^{+} 5 p^{5}\left({ }^{2} P_{1 / 2},{ }^{2} P_{3 / 2}\right)+e^{-}$.

Due to spin-orbit interaction, the final ionic states show a fine-structure splitting, the ${ }^{2} P_{1 / 2}$-state lying $1.31 \mathrm{eV}$ higher than the ${ }^{2} P_{3 / 2}$ ionic ground state. Two sorts of photoelectrons with different kinetic energies are therefore produced, which show up as two peaks in the photoelectron spectrum (see Fig. 5).

For these two ensembles of photoelectrons, corresponding to the two final ionic states, the spinpolarization parameters $\alpha, A$ and $\xi$ have been measured at several photon wavelengths between $90 \mathrm{~nm}$ and $48 \mathrm{~nm}$ via $A(\theta)$ and $P_{1}(\theta)$ as described in Chap. 2. The results, partly already published elsewhere [55], are shown as circles in Fig. 7. Important contributions to the error bars shown are the single statistical error of the spin-polarization analysis, the error in the determination of the light polarization and the uncertainty in the analyzing power of the Mott detector.

Results for the spin-parameter $\xi$ from measurements with unpolarized and linearly polarized light at resonance wavelengths $[23,25,26]$ are also shown as squares. The agreement with the data ob- 




A


Fig. 7. Spin-polarization parameters as function of the wavelength in the continuous range for photoelectrons leaving the xenon ion in the ${ }^{2} P_{1 / 2}$ and ${ }^{2} P_{3 / 2}$ state, respectively. Circles, this work and [55]; squares, experimental results from $[23,26]$; full curves, RRPA-calculation [41]; dashed curves, RPAE-calculation [39]

tained by means of circularly polarized synchrotron radiation is very good and demonstrates the validity of the spin-polarization formulae in Sect. 2 for different states of light polarization.

The quantity $A$ shown in Fig. 7 is different from the angle-integrated spin-polarization measured in previous experiments, where the Fano-effect for $\mathrm{Xe} 5 p^{6}$ has been investigated in the open continuum with circularly polarized synchrotron radiation [26]: In these older experiments, all photoelectrons produced were collected by means of an electric field, and the spin-polarization measured was the mean of the values for $A_{3 / 2}$ and $A_{1 / 2}$, weighted with the corresponding partial cross sections [57]. This mean value is close to but not equal to zero, due to the influence of the spin-orbit interaction on the continuum states.

For comparison, theoretical results for the spinparameters are also shown in Fig. 7. The solid curve is based on a relativistic random-phase approximation (RRPA) [41], the dashed curve depicts results calculated in non-relativistic RPA (RPAE) [39], both taking into account exchange interaction and correlations between the $5 p^{6}, 5 s^{2}$ and $4 d^{10}$ subshells. A recent calculation for the spin-parameter $\xi$ in relativistic time-dependent local density approximation (RTDLDA) [47] yields values between the RRPA- and the RPAE-results. The results of the three calculations agree very well with each other and with the experimental data.

\subsection{Determination of Transition Matrix Elements}

Photoionization of xenon atoms (ground state ${ }^{1} S_{0}$ ) in the $5 p$-shell reaches five final states with $J=1$ due to the dipole selection rules. These are called loosecoupling states or dissociation channels $i$. They can be characterized by the quantum numbers for the system ion + photoelectron [57]:

$$
\begin{aligned}
& i=\begin{array}{llll}
1 & 2 & 3
\end{array} \\
& \left(\begin{array}{llll}
\left({ }^{2} P_{3 / 2} d_{5 / 2}\right)_{1} & \left({ }^{2} P_{3 / 2} d_{3 / 2}\right)_{1} & \left({ }^{2} P_{3 / 2} s_{1 / 2}\right)_{1}
\end{array}\right. \\
& i=4 \quad 5 \\
& \left({ }^{2} P_{1 / 2} d_{3 / 2}\right)_{1} \quad\left({ }^{2} P_{1 / 2} s_{1 / 2}\right)_{1} .
\end{aligned}
$$

These transitions are characterized by five energy dependent reduced transition matrix elements $\tilde{D}_{i}$, which in general are complex numbers $D_{i} \mathrm{e}^{i \delta_{i}}$ with amplitude and phase. States corresponding to the same final ionic state are energetically degenerate and can interfere with each other. Since photoelectrons belonging to different final ionic states are experimentally separated, the coherence of channels with different ionic states is destroyed and a phase difference between them is not defined.

Since phase shifts of wavefunctions always appear as differences, the photoionization of $\mathrm{Xe} 5 p^{6}$ into the channels 1,2 and 3 is completely quantitatively characterized by the five real quantities $D_{1}$, $D_{2}, D_{3}, \delta_{1}-\delta_{2}$ and $\delta_{1}-\delta_{3}$. For the channels 4 and 5 with the final ionic state ${ }^{2} P_{1 / 2}$ the three real quantities $D_{4}, D_{5}$ and $\delta_{4}-\delta_{5}$ are already sufficient. All observable parameters which determine the photoionization of $\mathrm{Xe} 5 p^{6}$, can be expressed as functions of these five and three quantities, respectively. Vice versa, five and three independent observables, respectively, may represent a "complete" set of parameters, which allows the determination of all the matrix elements and phase-shift differences mentioned above. Such a complete analysis is given in this paper on the basis of the measured spin-parameters together with the existing data for the photoionization cross section $Q$ and the asymmetry parameter $\beta$. 
Table 1. The dynamical parameters, i.e. cross section $Q$, branching ratio $\rho$, angular asymmetry parameter $\beta$, and spin parameters $\xi$, $A$, and $\alpha$, as functions of matrix elements $D_{i}$ and phase-shift differences $\delta_{i}-\delta_{j}, \alpha, a_{0}$ and $\omega$ are the fine-structure constant, the Bohr radius, and the photon energy in atomic units $(1 \mathrm{a} . u=2 \mathrm{Ry})$, respectively

$$
\begin{aligned}
& Q=4 \pi^{2} \alpha a_{0}^{2} \omega \sum_{i=1}^{5} D_{i}^{2} \quad(10) \quad \xi_{1 / 2}=\frac{3 \sqrt{2} D_{4} D_{5} \sin \left(\delta_{5}-\delta_{4}\right)}{4\left(D_{4}^{2}+D_{5}^{2}\right)} \\
& \rho=\frac{D_{1}^{2}+D_{2}^{2}+D_{3}^{2}}{D_{4}^{2}+D_{5}^{2}}=\frac{Q_{3 / 2}}{Q_{1 / 2}} \\
& \beta_{1 / 2}=\frac{D_{4}^{2}+2 \sqrt{2} D_{4} D_{5} \cos \left(\delta_{5}-\delta_{4}\right)}{D_{4}^{2}+D_{5}^{2}} \\
& A_{1 / 2}=\frac{D_{5}^{2}-0.5 D_{4}^{2}}{D_{4}^{2}+D_{5}^{2}} \\
& \alpha_{1 / 2}=\frac{-D_{4}^{2}+\sqrt{2} D_{4} D_{5} \cos \left(\delta_{5}-\delta_{4}\right)}{D_{4}^{2}+D_{5}^{2}} \\
& \beta_{3 / 2}=\frac{1}{5} \frac{4 D_{1}^{2}-4 D_{2}^{2}-6 \sqrt{5} D_{1} D_{3} \cos \left(\delta_{3}-\delta_{1}\right)+6 D_{1} D_{2} \cos \left(\delta_{2}-\delta_{1}\right)-2 \sqrt{5} D_{3} D_{2} \cos \left(\delta_{3}-\delta_{2}\right)}{D_{1}^{2}+D_{2}^{2}+D_{3}^{2}} \\
& \xi_{3 / 2}=\frac{1}{20} \frac{15 D_{1} D_{2} \sin \left(\delta_{1}-\delta_{2}\right)+3 \sqrt{5} D_{2} D_{3} \sin \left(\delta_{2}-\delta_{3}\right)+6 \sqrt{5} D_{1} D_{3} \sin \left(\delta_{3}-\delta_{1}\right)}{D_{1}^{2}+D_{2}^{2}+D_{3}^{2}} \\
& A_{3 / 2}=\frac{1}{10} \frac{7 D_{1}^{2}-5 D_{3}^{2}-2 D_{2}^{2}-12 D_{1} D_{2} \cos \left(\delta_{2}-\delta_{1}\right)}{D_{1}^{2}+D_{2}^{2}+D_{3}^{2}} \\
& \alpha_{3 / 2}=\frac{1}{10} \frac{4 D_{1}^{2}-4 D_{2}^{2}+6 D_{1} D_{2} \cos \left(\delta_{2}-\delta_{1}\right)+10 \sqrt{5} D_{3} D_{2} \cos \left(\delta_{3}-\delta_{2}\right)}{D_{1}^{2}+D_{2}^{2}+D_{3}^{2}}
\end{aligned}
$$

The dependences of the experimental parameters upon the matrix elements $D_{i}$ and phase-shift differences $\delta_{i}-\delta_{j}$ were derived from the general relations given by Huang [36] and are given in Table 1 for the cross section $Q$ for photoionization in the $5 p$ shell, the branching ratio $\rho$ (ratio of the cross sections $Q_{3 / 2}$ and $Q_{1 / 2}$ for ionization with the final states $\mathrm{Xe}^{+}{ }^{2} P_{3 / 2}$ and $\mathrm{Xe}^{+2} P_{1 / 2}$ ), the asymmetry parameters $\beta_{3 / 2}$ and $\beta_{1 / 2}$ of the differential cross section and for the spin-polarization parameters $\xi_{3 / 2}$, $\xi_{1 / 2}, A_{3 / 2}, A_{1 / 2}, \alpha_{3 / 2}$ and $\alpha_{1 / 2}$. These relations were independently checked using the density matrix formalism [72]. Only the cross section $Q$ depends on the absolute values of the matrix elements, all other parameters contain only ratios of matrix elements. Like the cross section and the branching ratio, the parameter $A_{1 / 2}$ contains no phase-shift difference, and $A_{3 / 2}$ contains only the phase-shift difference $\delta_{2}-\delta_{1}$ between $d$ partial waves.

From $Q$ and $\rho$, the partial cross sections $Q_{3 / 2}$ and $Q_{1 / 2}$ can be calculated. For each of the two final ionic states ${ }^{2} P_{3 / 2}$ and ${ }^{2} P_{1 / 2}$ five measured quantities can thus be used to determine $D_{1}, D_{2}, D_{3}, \delta_{2}-\delta_{1}$ and $\delta_{3}-\delta_{1}$, as well as $D_{4}, D_{5}$ and $\delta_{4}-\delta_{5}$, respectively. The third phase-shift difference can be calculated as $\delta_{2}-\delta_{3}=\left(\delta_{2}-\delta_{1}\right)-\left(\delta_{3}-\delta_{1}\right)$. The values for $Q$ and $\beta$ were taken from the literature by comparison of data given by several authors. The existing cross section measurements [73-82] are partly critically reviewed by West and Morton [83]. For the branching ratio $\rho$, recent measurements with synchrotron radiation [84-89] near the ionization threshold complete former experimental results [90-97].
Excellent data exist for the asymmetry parameter $\beta$, which has been measured at resonance wavelengths of discharge sources $[25,98-107]$ as well as with synchrotron radiation $[87-89,108,109]$. In addition to the spin-parameters $A, \alpha$ and $\xi$ measured with circularly polarized synchrotron radiation, $\xi$-values from experiments with unpolarized line radiation $[23,26]$ were used (at the $\mathrm{HeI}(21.22 \mathrm{eV})$ and $\mathrm{NeIb}$ $(16.85 \mathrm{eV})$ resonance lines).

For the final ionic state $\mathrm{Xe}^{+2} P_{1 / 2}$, the three unknown quantities $D_{4}, D_{5}$ and $\delta_{4}-\delta_{5}$ can be determined from experimental data for five photoionization parameters, namely $Q_{1 / 2}, \beta_{1 / 2}, \alpha_{1 / 2}, A_{1 / 2}$, and $\xi_{1 / 2}$. Three independent solutions could therefore be derived, which are identical within the error limits and are shown as filled circles as function of the photon energy in Fig. 8. Since for this final state three suitable measured parameters are already sufficient to determine the matrix elements and the phase difference, this analysis could already be performed earlier on the basis of results for $Q_{1 / 2}, \beta_{1 / 2}$ and $\xi_{1 / 2}$ at several resonance wavelengths [57]. These earlier results are shown as squares in Fig. 8 and are in excellent agreement with the present values obtained from measurements with circularly polarized synchrotron radiation. This is valuable evidence for the consistency of our experimental data and for the proper function of the new apparatus. We have plotted the quantum-defect difference $\mu_{4}-\mu_{5}$ instead of the phase-shift difference, i.e. the Coulomb-phase shift has been subtracted (cf. Eq. (21)). The curves shown represent theoretical results and are explained in Chap. 4.3. 


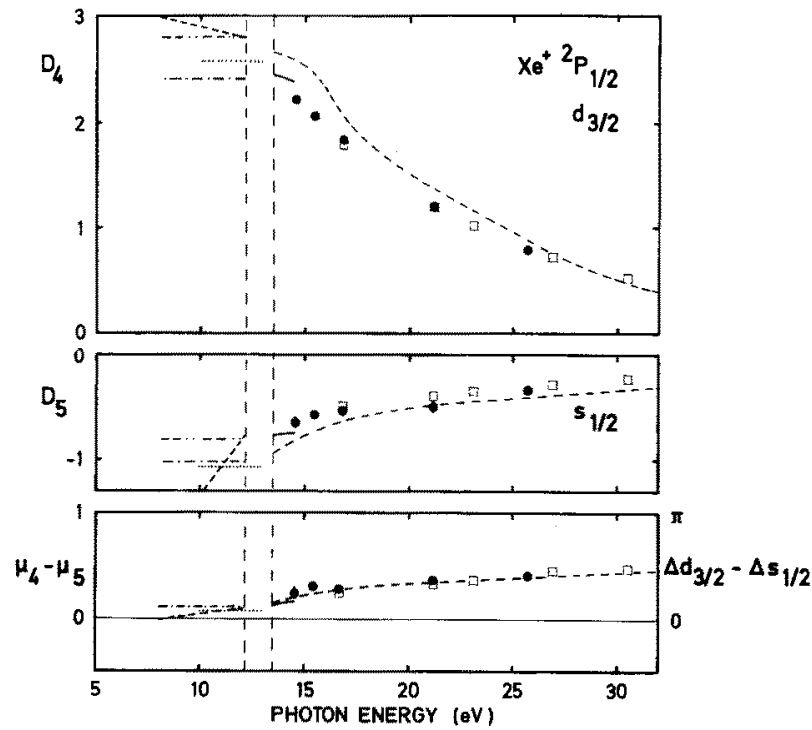

Fig. 8. Matrix elements $D_{i}$ and quantum-defect differences $\mu_{i}-\mu_{j}$ for photoionization with the final ionic state $\mathrm{Xe}^{+2} P_{1 / 2}$ as function of the photon energy in the continuum region. The vertical dashed lines indicate the ionization thresholds. In the continuum region are shown: Circles, this work; squares, results from [57]; dashed curve, RPAE-calculation [119]; full curve, calculated on the basis of RRPA-MQDT parameters from [52]. The curves in the discrete region are calculated from MQDT-parameters given by the following authors (cf. [57]): dashed, Lee and Dill [115]; dotted, Geiger [113]; double-dashed-dotted, Geiger [118]; dashed-dotted, Geiger [114]

For the final ionic state $\mathrm{Xe}^{+2} P_{3 / 2}$, the matrix elements and phase-shift differences could up to now only be approximately determined [57], since the number of measured photoionization parameters was not sufficient. In that approximation, the difference $\delta_{1}-\delta_{2}$ between the $d$-channels was assumed to vanish [57]. With the new experimental data, however, the five quantities $D_{1}, D_{2}, D_{3}, \delta_{1}-\delta_{3}$ and $\delta_{1}-\delta_{2}$ can be determined without any approximation. The results are shown as filled circles in Fig. 9.

We note that the underlying system of nonlinear equations (cf. Table 1) does not necessarily have only one unambiguous mathematical solution. There exist, however, additional constraints: the quantumdefect differences must be smooth functions of photon energy, and the "sign convention" for the reduced matrix elements determines $D_{1}, D_{2}, D_{3}$ and $D_{4}$ to be positive and $D_{5}$ to be negative at the ionization threshold $[57,72]$. These conditions rule out those solutions which are physically unreasonable. The error bars in Figs. 8 and 9 result from the errors of all experimental data involved, containing the single statistical error of spin-polarization values.

\subsection{Discussion in the Context of Multichannel Quantum Defect Theory}

The results presented in Sect. 4.2 can be compared with data in the discrete spectral range in the frame-

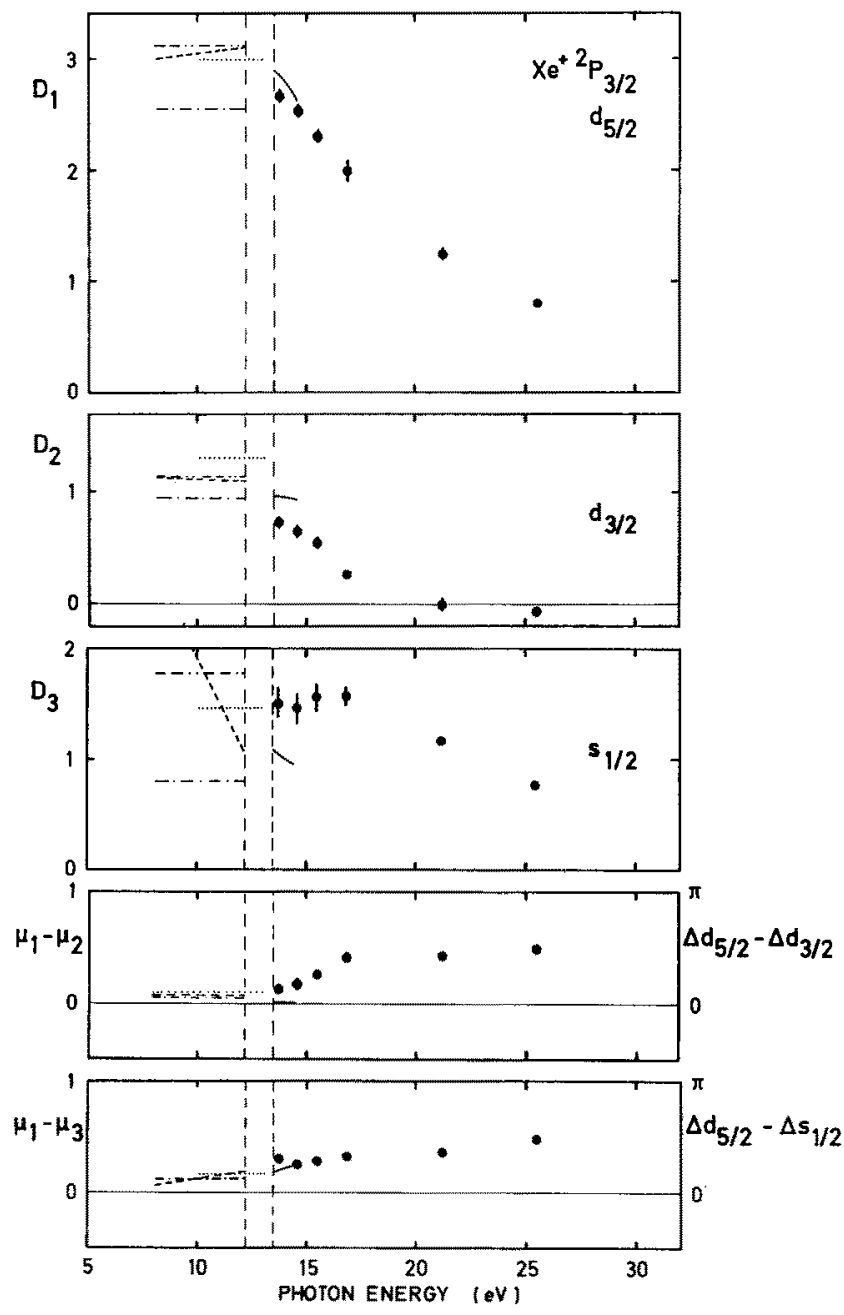

Fig. 9. Matrix elements $D_{i}$ and quantum-defect differences $\mu_{i}-\mu_{j}$ for photoionization with the final ionic state $\mathrm{Xe}^{+2} P_{3 / 2}$ as function of the photon energy in the continuum region. The meaning of the symbols is the same as in Fig. 8

work of Multichannel Quantum Defect Theory (MQDT). The MQDT describes a multitude of quantities near the ionization threshold of an atomic system, such as the positions and intensities of spectral lines as well as profiles of autoionization resonances and photoionization cross sections, in terms of a small set of only weakly energy dependent parameters $[1,33,110-112]$. MQDT connects the loosecoupling states (dissociation channels i) described above with the close-coupling states or eigenchannels $\alpha$, which for discrete excitation can be classified by LS quantum numbers. For dipole transitions from the ground state $\mathrm{Xe} 5 p^{6}\left({ }^{1} S_{0}\right)$, we adopt the following notation for the eigenchannels:

$$
\begin{aligned}
& \alpha=\begin{array}{ccc}
\alpha & 2 & 3 \\
p^{5} d\left({ }^{3} P_{1}\right) & p^{5} d\left({ }^{3} D_{1}\right. & p^{5} d\left({ }^{1} P_{1}\right) \\
\alpha= & 4 & 5 \\
p^{5} s\left({ }^{3} P_{1}\right) & p^{5} s\left({ }^{1} P_{1}\right) .
\end{array}
\end{aligned}
$$


Eigenchannels and dissociation channels are connected by the transformation $U_{i \alpha}$ according to:

$D_{i} \mathrm{e}^{\mathrm{i} \pi \mu_{i}}=\sum_{\alpha} U_{i \alpha} D_{\alpha} \mathrm{e}^{\mathrm{i} \pi \mu_{\alpha}}$

The transition amplitudes $D_{\alpha}$, which give the intensity, and the eigenquantum defects $\mu_{\alpha}$, which give the energetic position of spectral lines, are thus transformed into the modified quantities $D_{i}$ and $\mu_{i}$ by means of the matrix $U_{i \alpha}$. Several authors have calculated $U_{i \alpha}$ for xenon, both ab initio [52] or semiempirically from spectroscopic data [113-115]. Like the eigenquantum defects, $U_{i x}$ does not depend or depends only weakly on photon energy. In the continuous spectral range, the quantities $D_{i}$ are identical to the amplitudes of the reduced matrix elements defined in Sect. 4.2, and the modified eigenquantum defects $\mu_{i}$ are related to the phase shifts $\delta_{i}$ of the continuum wavefunctions [54]:

$\delta_{i}=\sigma_{l}+\pi \mu_{i}-\pi \frac{l}{2}$

$\sigma_{i}$ is the phase shift for an outgoing partial wave with angular momentum $l$ in a pure Coulomb-field, which also appears in electron scattering at the ion as the well-known Coulomb phase shift. It can be analytically calculated [116]:

$\sigma_{i}=\arg \Gamma\left(l+1-i \varepsilon^{-1 / 2}\right)$.

A helpful formula is [117]:

$\sigma_{l}-\sigma_{l^{\prime}}=\sum_{v=l+1}^{l^{\prime}} \operatorname{arctg} \frac{1}{v \sqrt{\varepsilon}}$.

$\varepsilon$ is the kinetic energy of the photoelectron measured in Rydbergs. The additional phase shift $\pi \mu_{i}=\Delta_{i}$ is due to the deviation of the true potential from a pure Coulomb potential. The term $\pi l / 2$ satisfies the sign convention for the matrix elements.

For comparison with the matrix elements and quantum-defect differences in the continuous spectral region, Figs. 8 and 9 show the corresponding quantities in the discrete spectral range, which were calculated in [57] according to Eq. (20) from the MQDT-parameters $D_{\alpha}, \mu_{\alpha}$ and $U_{i \alpha}$ given by different authors. The dotted curves are based on the analysis by Lee and Dill [115], where the quantum-defect parameters are independent of photon energy. The other straight lines originate from Geiger and are based on data from energy-loss spectroscopy of fast electrons $[113,114]$. The dashed lines were calculated with energy independent $U_{i \alpha}$ and energy dependent $D_{\alpha}$ and $\mu_{\alpha}$ [113], whereas for the doubledashed-dotted lines the same $U_{i \alpha}$ and $\mu_{\alpha}$, but energy independent $D_{\alpha}$ were used [118]. The dashed-dotted lines employ completely energy independent MQDT-parameters [114].

In the continuous range near the ${ }^{2} P_{1 / 2}$-threshold the full curves represent results based on MQDTparameters, which were calculated $a b$ initio in RRPA [52]. We have assumed $U_{i a}$ to be energy independent (values are published for the ionization threshold only), the other parameters are weakly energy dependent. The dashed curve in the continuous range in Fig. 8 represents the matrix elements and quantum-defect differences calculated $a b$ initio in RPAE [119]. The corresponding data for the RRPA-calculations [41] are not available in the literature.

The data in the continuum smoothly match the values in the discrete in the region of the ionization thresholds. This especially holds for the quantumdefect differences. Linear extrapolation from the discrete into the continuum, however, is only possible to a certain extent. The energy dependence of the quantum defects $\mu_{4}-\mu_{5}$ and $\mu_{1}-\mu_{3}$ is well reproduced, in contrast to the absolute values of the matrix elements, which strongly decrease with photon energy. In particular, the phase difference $\mu_{1}-\mu_{2}$ between the $d$-waves $d_{5 / 2}$ and $d_{3 / 2}$, which is small in the discrete range, shows a pronounced energy dependence in the continuum. This means that the phases of the two partial waves with equal angular momentum strongly depend upon whether spin and angular momentum in the continuum are parallel or antiparallel. This is a manifestation of spin-orbit interaction in the continuum, independent of ground state or final ionic state fine-structure splitting. The amplitudes of the transition matrix elements are also influenced by this interaction. When we look at the non-relativistic model, where spin-orbit interaction in the continuum is neglected, there only exist transitions into pure $d$ - and $s$-continua, with transition matrix elements $D_{d}$ and $D_{s}[72]$ :

$D_{1}=3 \sqrt{\frac{1}{10}} D_{d} ; \quad D_{2}=\sqrt{\frac{l}{10}} D_{d} ; \quad D_{3}=D_{s}$

and $\delta_{1}=\delta_{2}=\delta_{d}, \delta_{3}=\delta_{s}$. In particular, the relation $D_{1}=3 D_{2}$ holds. This condition is approximately true at the ionization threshold, but completely breaks down about $5 \mathrm{eV}$ above threshold, where $D_{2}$ goes through zero (cf. Fig. 9). The spin-orbit interaction in the continuum thus shows up drastically in the amplitudes and in the relative phases of transition matrix elements. One might have the impression that the zero-crossing of $D_{2}$ (Cooper minimum) and the corresponding strong increase of the $d$-phase difference $\mu_{1}-\mu_{2}$ are correlated to each other which would mean that $D_{2}$ and its phase $\mu_{2}$ are not inde- 
pendent quantum-mechanical quantities. The following Chap. 4.4 tries to give an answer to this question using an other classification scheme for the states involved.

\subsection{Discussion in the Context of the Angular-Momentum-Transfer Classification}

The classification of the loose-coupling states by orbital and total angular momentum quantum numbers is the traditional but, of course, only one of several possible schemes. An alternative approach is the angular-momentum-transfer formalism, first applied by Dill and Fano [115, 120-125], where the angular momentum $\mathbf{j}_{\gamma}$ of the ionizing photon and the orbital angular momentum $\mathbf{I}$ of the photoelectron are related to the angular-momentum transfer t:

$\mathbf{t}=\mathbf{j}_{\mathrm{y}}-\mathbf{l}$.

Two classes of transitions are introduced, those for which the transfer of parity matches the parity of the momentum transfer:

$\pi_{0} \pi_{f}=(-1)^{t}, \quad l=t \pm 1$

the so-called "parity-favoured" transitions, and those for which the transfer of parity does not match the parity of the momentum transfer:

$\pi_{0} \pi_{f}=-(-1)^{t}, \quad l=t$

the so-called "parity-unfavoured" transitions. $\pi_{0}$ is the parity of the atom, $\pi_{f}$ the parity of the photoion. *

For the photoionization of $\mathrm{Xe} 5 p^{6}$ with the final state $\mathrm{Xe}^{+2} P_{3 / 2}$, there exist two parity favoured transitions with $t=1$ into the $s$ - and $d$-continua, described by the complex transition matrix elements $\tilde{S}_{3}$ and $\tilde{S}_{1}$, and one parity-unfavoured $d$-channel with $t$ $=2$ and matrix element $\tilde{S}_{2}$ [34]. For $\mathrm{Xe}^{+2} P_{1 / 2}$, there are only parity-favoured transitions with $t=1$; the transition matrix elements are identical with $\tilde{D}_{4}$ and $\tilde{D}_{5}$ discussed in Sect. 4.2 [34].

We therefore focus on $\tilde{S}_{1}, \tilde{S}_{2}$ and $\tilde{S}_{3}$, which are connected with the matrix elements $\tilde{D}_{1}, \tilde{D}_{2}$ and $\tilde{D}_{3}$ by the transformation $[72,113,115]$ :

$\tilde{S}_{1}=\sqrt{\frac{9}{10}} D_{1} \mathrm{e}^{\mathrm{i}\left(\delta_{1}-\delta_{3}\right)}+\sqrt{\frac{1}{10}} D_{2} \mathrm{e}^{\mathrm{i}\left(\delta_{2}-\delta_{3}\right)}$

$\tilde{S}_{2}=\sqrt{\frac{1}{10}} D_{1} \mathrm{e}^{\mathrm{i}\left(\delta_{1}-\delta_{3}\right)}-\sqrt{\frac{9}{10}} D_{2} \mathrm{e}^{\mathrm{i}\left(\delta_{2}-\delta_{3}\right)}$

$\tilde{S}_{3}=D_{3}$.

* To avoid confusion, we note that parity-unfavoured transitions have no connection with parity violating processes
The relative phases of $\tilde{S}_{1}$ and $\tilde{S}_{2}$ refer to $\tilde{S}_{3}$. In the non-relativistic approximation, where $D_{1}=3 D_{2}$ and $\delta_{1}=\delta_{2}, \tilde{S}_{2}$ vanishes, and $\tilde{S}_{1}$ and $\tilde{S}_{3}$ are matrix elements for transitions into pure $d$ - and $s$-continua, respectively. In general, the parity-unfavoured contributions originate from anisotropic interactions between photoelectron and ion $[120,123]$. In this special case, $\tilde{S}_{2}$ can be considered to be a measure for the deviation of the real conditions from the nonrelativistic approximation. Thus, $\tilde{S}_{2}$ should visualize the influence of spin-orbit interaction in the continuum. This is indeed the case, as can be seen, when the Coulomb-phase is eliminated:

$\tilde{S}_{1}=S_{1} \mathrm{e}^{\mathrm{i}\left(\phi_{1}-\phi_{3}\right)} \mathrm{e}^{\mathrm{i}\left(\sigma_{d}-\sigma_{s}-\pi\right)}$

$\tilde{S}_{2}=S_{2} \mathrm{e}^{\mathrm{i}\left(\phi_{2}-\phi_{3}\right)} \mathrm{e}^{\mathrm{i}\left(\sigma_{d}-\sigma_{s}-\pi\right)}$

$\tilde{S}_{3}=S_{3}=D_{3}$.

In analogy to Eq. (21), the quantities:

$\eta_{1}-\eta_{3}=\left(\phi_{1}-\phi_{3}\right) / \pi$

$\eta_{2}-\eta_{3}=\left(\phi_{2}-\phi_{3}\right) / \pi$

may be interpreted as modified quantum-defect differences. The real transition amplitudes and the quantum-defect differences are shown in Fig. 10 as functions of the photon energy. The parity-unfavoured amplitude $S_{2}$ is clearly different from zero and amounts to about one half of the parity-favoured amplitudes for photon energies above $20 \mathrm{eV}$. Considerable contributions to the transition probability into the $\mathrm{Xe}^{+2} P_{3 / 2}$ final state are thus due to the parity-unfavoured channel.

While $S_{1}$ and $S_{3}$ decrease with photon energy (as all $D_{i}$ do), $S_{2}$ goes through a maximum at about $5 \mathrm{eV}$ above the ionization threshold. To our knowledge, there exist no systematic studies of the behaviour of angular-momentum-transfer matrix elements as function of the photon energy. We only point out that the maximum in $S_{2}$ occurs in the region where $\mathrm{D}_{2}$ goes through zero, without claiming that there is a deeper reason for a fact which might well be a mere coincidence.

When the classification by total angular momentum is used, the spin-orbit interaction in the continuum influences the amplitudes as well as the phases of the transition matrix elements. This seems to be different in the angular-momentum-transfer formalism. Figure 11 shows all quantum-defect differences between $d$ - and $s$-continua, revealing the striking equality of $\mu_{1}-\mu_{3}$ and $\eta_{1}-\eta_{3}$, which belong to the ${ }^{2} P_{3 / 2}$ ionic state, with $\mu_{4}-\mu_{5}$, which belongs to the ${ }^{2} P_{1 / 2}$ ionic state. The difference $\mu_{2}-\mu_{3}$, however, deviates strongly from these common values for photon energies above $17 \mathrm{eV}$, whereas $\eta_{2}-\eta_{3}$ does 

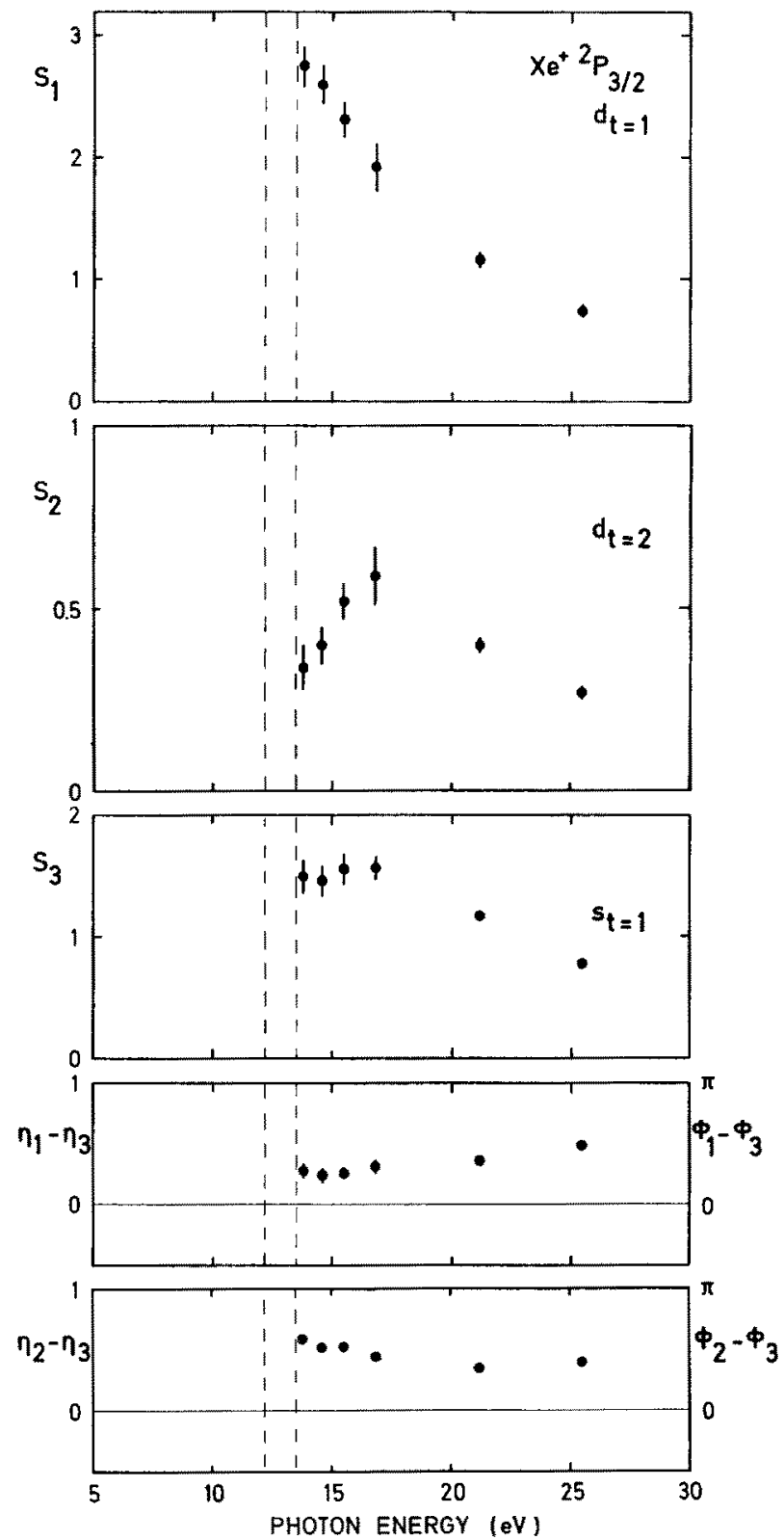

Fig. 10. Matrix elements $S_{i}$ and quantum-defect differences $\eta_{i}-\eta_{j}$ for photoionization with the final ionic state $\mathrm{Xe}^{+2} P_{3 / 2}$ as function of the photon energy in the continuum region. Transitions are classified by angular-momentum transfers. The vertical dashed lines indicate the ionization thresholds

not. In this energy region, all the quantum-defect differences in the angular-momentum-transfer formalism between $d$ - and s-continua are identical in good approximation, independent of the final ionic state and of the angular-momentum transfer. In this classification scheme, the influence of spin-orbit interaction in the continuum is thus mainly described by the parity-unfavoured amplitude $S_{2}$, which vanishes when the continuum spin-orbit interaction vanishes; furthermore the number of quantum-mechani-

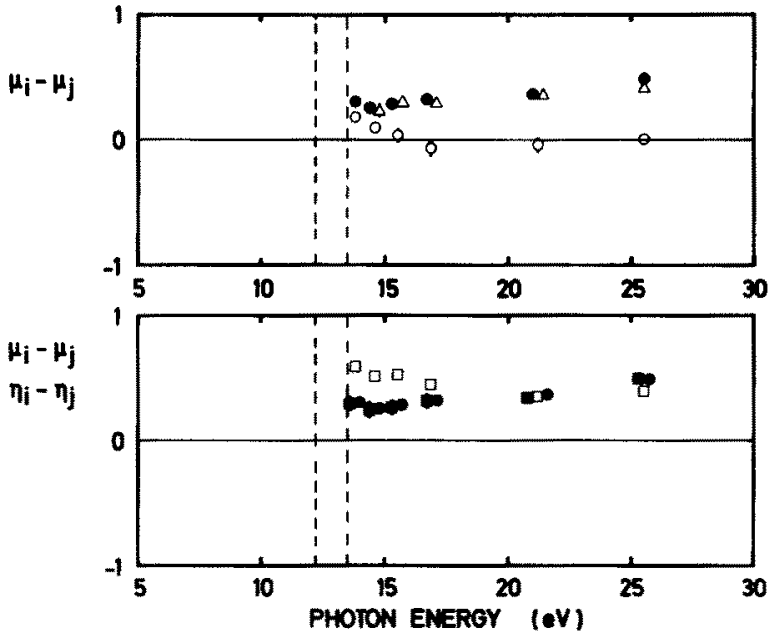

Fig. 11. The quantum-defect differences between $d$ - and $s$-continua.

•: $\mu_{1}-\mu_{3}{ }^{2} P_{3 / 2}\left(d_{5 / 2}-s_{1 / 2}\right)$

$\Delta: \mu_{4}-\mu_{5}{ }^{2} P_{1 / 2}\left(d_{3 / 2}-s_{1 / 2}\right)$

o: $\mu_{2}-\mu_{3}{ }^{2} P_{3 / 2}\left(d_{3 / 2}-s_{1 / 2}\right)$

: $\eta_{2}-\eta_{3}{ }^{2} P_{3 / 2}\left(d_{t=2}-s_{t=1}\right)$

-: $\eta_{1}-\eta_{3}{ }^{2} P_{3 / 2}\left(d_{t=1}-s_{t=1}\right)$

The vertical dashed lines indicate the ionization thresholds

cal quantities needed for the complete characterization of the photoionization process may be said to be reduced by one since the phase-shift differences are equal.

\section{The Autoionization Region}

\subsection{Experimental Results}

In the wavelength region between the two first ionization thresholds of xenon at $102.2 \mathrm{~nm}$ and $92.2 \mathrm{~nm}$ (autoionization range), only the dissociation channels belonging to the $\mathrm{Xe}^{+2} P_{3 / 2}$ final state are open. In the same wavelength range, discrete excited states are possible (a $5 p \rightarrow n s$ and a $5 p \rightarrow n d$ Rydberg series converging to the ${ }^{2} P_{1 / 2}$-threshold), which can decay into the continuum. This gives rise to two series of autoionization resonances in the photoionization cross section, a series of sharp s-resonances and a series of broad $d$-resonances. Theses features were first observed in 1935 by H. Beutler [126] at the Fritz-Haber-Institute in Berlin and later explained theoretically by Fano $[127,128]$. Resonance structures were not only found for the cross section $Q$ $[75,77-79,82,129,130]$, but also for the asymmetry parameter $\beta[131,132]$ and the spin-parameter $A$ $[15,26]$.

Figure 12 [56] shows all five photoionization parameters for the wavelength range from $100 \mathrm{~nm}$ to 


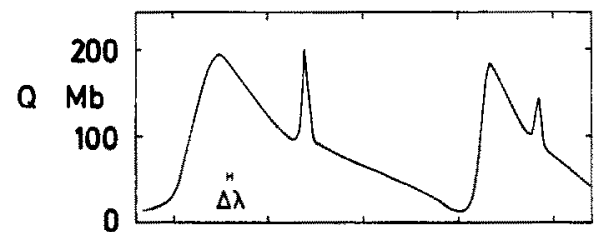

$\beta$
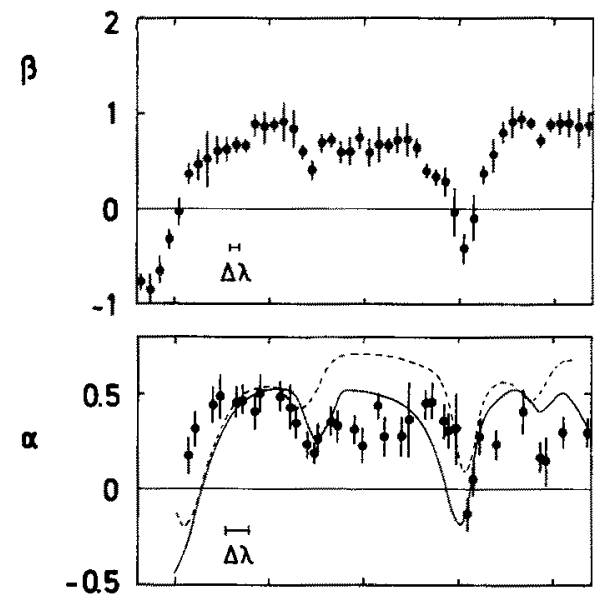

A

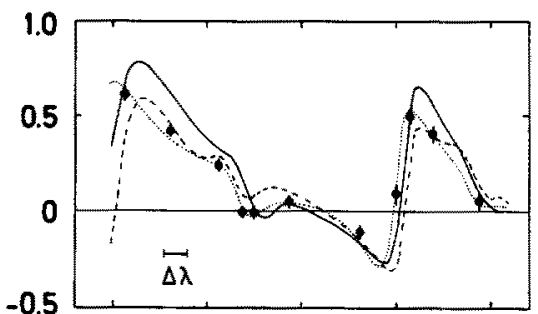

$\xi$

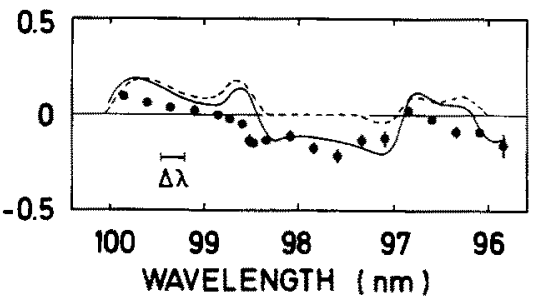

Fig. 12. Cross section $Q$ [82], asymmetry parameter $\beta$ [131] and spin-parameters $\alpha, A$ and $\xi$ for the $5 p$-autoionization of xenon (from [56]). For the spin-parameters, the symbols denote: Circles, experimental results; full curves, RRPA-calculation [52] (convoluted with $\Delta \lambda=0.25 \mathrm{~nm}$ ); dashed curves, MQDT-calculation [33] (convoluted with $A \lambda=0.25 \mathrm{~nm}$ ); dotted curve for $A$, based on experimental spin-polarization data of the angle-integrated photoelectron flux $[15,26]$, convoluted to correspond to the resolution of $\Delta \lambda=0.25 \mathrm{~nm}$

$96 \mathrm{~nm} . A, \alpha$ and $\xi$ were measured with the apparatus described in Chap. 3 with a resolution of $0.25 \mathrm{~nm}$ [56]. For $A$, there exist spin-polarization data of the total photocurrent $[15,26]$ with a resolution of $0.05 \mathrm{~nm}$, which were convoluted to match the resolution used in this work and are shown as a dotted curve in Fig. 12. Both sets of results for $A$ are in good agreement, which confirms the reliability of the data measured in this experimentally difficult region, where the photoelectrons have kinetic energies below $1 \mathrm{eV}$ (cf. Fig. 6).
Whereas $A$ and $\xi$ show a change of sign within the broad $d$-resonances near the minimum and in the decreasing wing of the cross section, the parameters $\alpha$ and $\beta$ only slightly vary through a resonance except for a dip near the cross-section minimum. The results for $A[15,26]$ and $\beta[131]$, measured with a bandwidth of $0.05 \mathrm{~nm}$ and $0.1 \mathrm{~nm}$, respectively, demonstrate that the narrow s-resonances influence the energy dependence of the dynamical parameters in the same way as the $d$-resonances do. In our measurements, however, the structure near the $s$ resonances is only partly resolved due to the bandwidth of $0.25 \mathrm{~nm}$.

Figure 12 also contains theoretical results for the spin-parameters. The full curves are based on abinitio RRPA-calculations [52], including correlations for $5 s-, 5 p$ - and $4 d$-electrons, the dashed curves represent the spin-parameters calculated by Lee [33] from semiempirical MQDT-parameters [133]. Both curves show the theoretical results, convoluted with the experimental bandwidth of $0.25 \mathrm{~nm}$.

The theoretical data agree remarkably well with the experimental results, both in the structure and in the absolute values of the spin-parameters, especially in view of the complexity of the process. We note, however, that the theoretical values for the broad $d$ resonance at about $99.5 \mathrm{~nm}$ are systematically shifted towards shorter wavelengths by approximately $0.3 \mathrm{~nm}$ with respect to the experimental values, although the position of the s-resonance in the photoionization parameters is very well reproduced by the calculations. This discrepancy concerning the relative position of $s$ - and $d$-resonances, which already appears in the spectral behaviour of $\beta$ [131] and $A$ $[15,26]$, is thus confirmed by the angle-resolved spin-polarization measurements. Johnson et al. [52] see the reason for this discrepancy in the limited correlation included in their RRPA-calculation. Further discrepancies between theoretical and experimental results appear for the spin-parameter $\xi$, which may be due to the fact that the expression for $\xi$ contains the sines of the phase-shift differences, whereas in the relations for the other spin-parameters and for $\beta$, only the cosine of the phase-shift differences appears. Small phase-shift differences may therefore have a large influence on $\xi$, while the other parameters are only slightly affected.

\subsection{Determination of Transition Matrix Elements}

The variation of the photoionization parameters across an autoionization resonance corresponds to structures in the transition matrix elements $[33,51$, $52,57,113]$. These structures can still be described 
by only weakly energy dependent MQDT-parameters $D_{\alpha}, \mu_{\alpha}$ and $U_{i \alpha}$, which correspond to the five eigenchannels $\alpha$ defined in 4.3. The connection between close-coupling and loose-coupling states, however, is much more complicated in the autoionization range than in the continuum [133]:

$D_{i} \mathrm{e}^{\mathrm{i} \pi \mu_{i}}=\sum_{\rho=1}^{3} \bar{T}_{i}^{\rho} \mathrm{e}^{\mathrm{i} \pi \tau_{\rho}} \bar{D}_{\rho}$.

$\tau_{\rho}$ are the scattering eigenphases of the three scattering eigenchannels $\rho, \bar{D}_{\rho}$ are the corresponding eigenamplitudes, and $\bar{T}_{i}^{\rho}$ is a transformation matrix connecting the scattering eigenchannels $\rho$ and the open dissociation channels $i=1,2,3$. The quantities $\tau_{\rho}, \bar{D}_{\rho}$ and $\bar{T}_{i}^{\rho}, \rho=1,2,3$, can be determined from the closecoupling quantities $\mu_{\alpha}, D_{\alpha}$ and $U_{i \alpha}, \alpha=1, \ldots, 5[113$, 115]. These weakly energy dependent quantities are thereby transformed into parameters varying strongly with energy, which according to (36) leads to the resonance structures in the transition matrix elements.

The formulae given in Sect. 4.2 for the connection between the observables $\beta, \alpha, A, \xi$ and the quantities $D_{1}, D_{2}, D_{3}$ and $\delta_{2}-\delta_{1}, \delta_{3}-\delta_{1}$ are still valid in the autoionization range. Since only the channels $i=1,2,3$ corresponding to the ${ }^{2} P_{3 / 2}$ final ionic state are open, the photoionization cross section is given by:

$Q=4 \pi^{2} \alpha a_{0}^{2} \omega \sum_{i=1}^{3} D_{i}^{2}$.

$a_{0}$ being the Bohr radius, $\alpha$ the fine-structure constant, and $\omega$ the photon energy in atomic units. In analogy to the procedure described in Sect. 4.2 the quantities $D_{1}, D_{2}, D_{3}$ and the quantum-defect differences $\mu_{1}-\mu_{2}, \mu_{1}-\mu_{3}$ can be determined from the measured values for $Q, \beta, A, \alpha$ and $\xi$.

First of all, the data for the cross section $Q$ [82] and the asymmetry parameter $\beta$ [131] were convoluted to correspond to a bandwidth of $0.25 \mathrm{~nm}$. These values were then used, together with the data for $A, \alpha$ and $\xi$ shown in Fig. 12, for the determination of the matrix elements $D_{i}$ and the phase-shift differences $\mu_{i}-\mu_{j}$. According to the transformations (28)-(30), the angular-momentum-transfer matrix elements $S_{1}, S_{2}$ and the quantum-defect differences $\eta_{1}-\eta_{3}$ and $\eta_{2}-\eta_{3}$ were calculated. The results are shown in Figs. 13 and 14 as function of the wavelength of the ionizing radiation.

The amplitudes $D_{1}$ and $S_{1}$ clearly reflect the wavelength dependence of the cross section. Near the maximum of the $d$-resonances, these amplitudes give the major contribution to the cross section. Near the minimum of the cross section, however, the

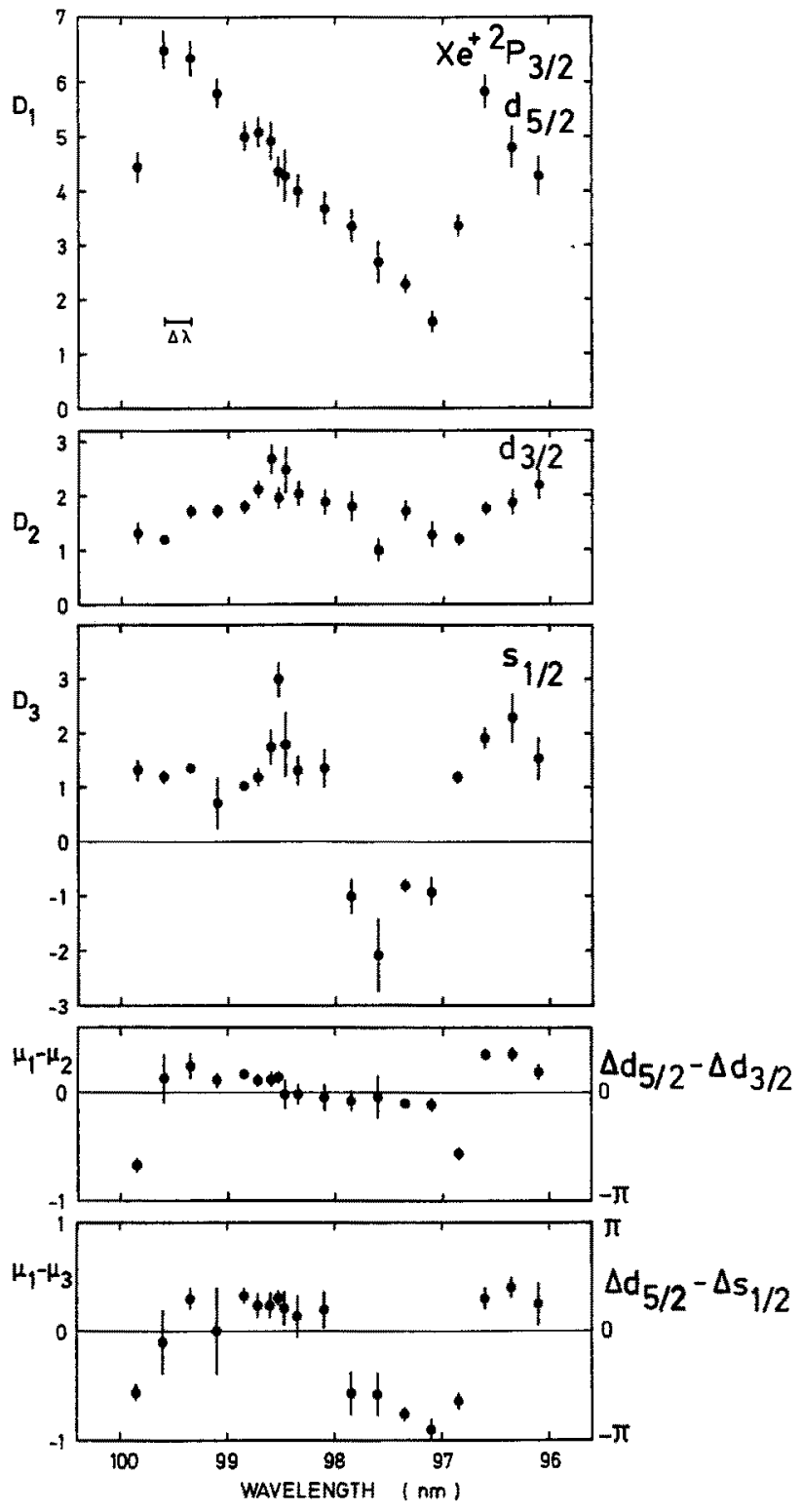

Fig. 13. Matrix elements $D_{i}$ and quantum-defect differences $\mu_{i}-\mu_{j}$ for photoionization of xenon in the $5 p$-autoionization range as function of the wavelength

amplitudes of the remaining channels nearly equal the amplitudes $D_{1}$ and $S_{1}$. This is the main reason for the characteristic minima in $\beta, \alpha$ and $A$. The wavelength dependence of the parameter $\xi$ is mainly caused by the variation of the quantum-defect differences. The zeros of $\xi$, for instance, are located near the zeros of the quantum-defect differences, since $\xi$ basically is a linear combination of terms proportional to the sines of the phase-shift differences.

Structures connected with the sharp $s$-resonances are largely washed out due to the experimental bandwidth of $0.25 \mathrm{~nm}$. The $s$-resonance at $98.5 \mathrm{~nm}$, however, clearly shows up in the amplitude $D_{3}$, only 

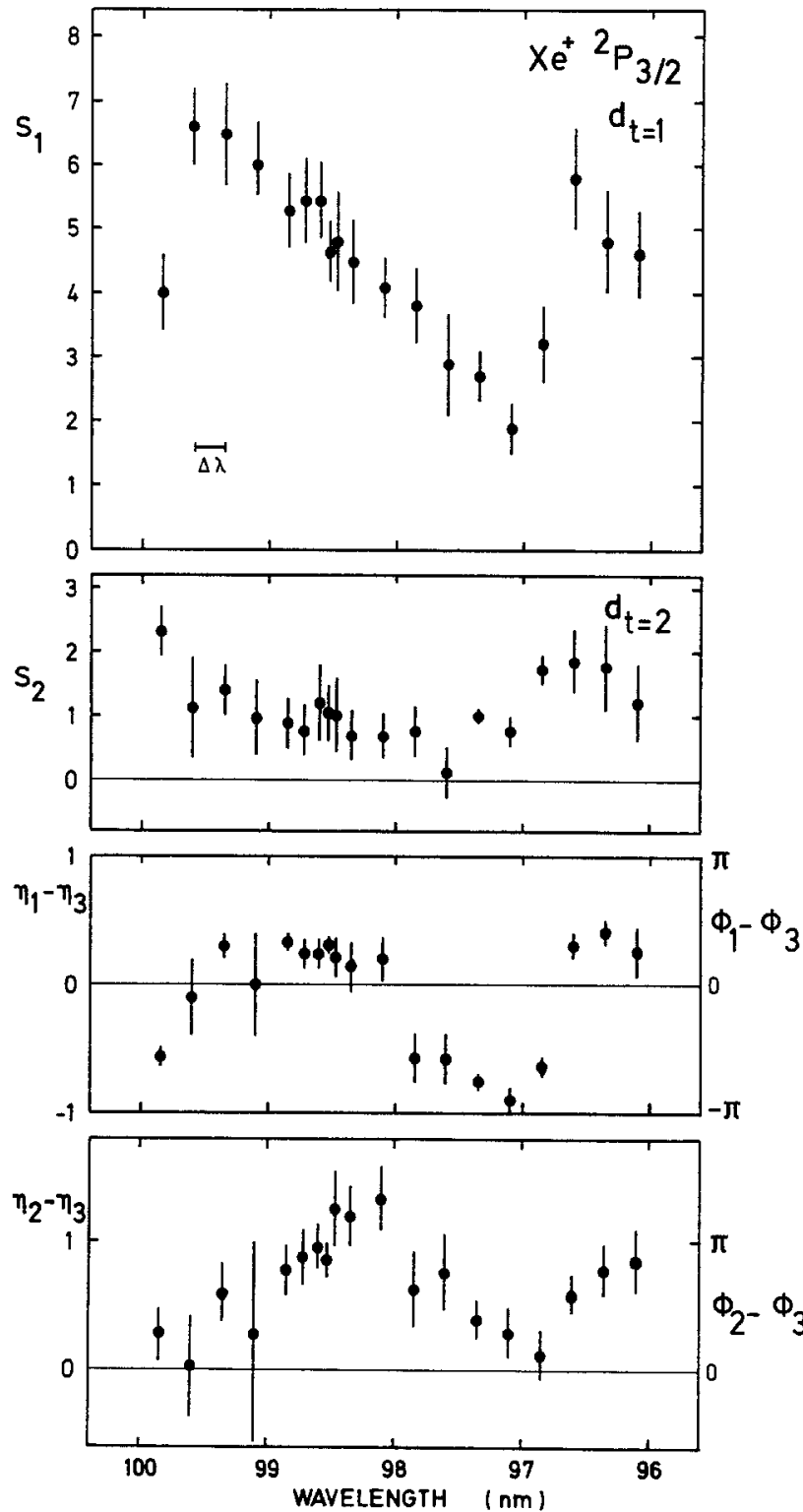

Fig. 14. Matrix elements $S_{i}$ and quantum-defect differences $\eta_{i}-\eta_{j}$ for photoionization of xenon in the $5 p$-autoionization range as function of the wavelength for classification of the transitions by angular-momentum transfers. Note that $S_{3}$ (not shown) is identical with $D_{3}$ given in Fig. 13

weakly in $D_{1}$ and $S_{1}$ but significantly also in $D_{2}$, the amplitude corresponding to the transition into the $d_{3 / 2}$-continuum. This appearance of the $s$-resonance in the $d_{3 / 2}$-transition in spite of the low experimental resolution is evidence of strong interchannel interaction between channels of different orbital angular momenta $[57,134]$. The relatively large values for the parity-unfavoured amplitude $S_{2}$, especially near the cross section minimum, further underline the importance of correlation effects in the autoionization region.

\section{Summary and Outlook}

Spin- and angle-resolved photoelectron spectroscopy with circularly polarized radiation in addition to measurements of the differential cross section exploits the full information about the photoionization process, which is carried by the outgoing photoelectrons. The three dynamical spin-parameters $A, \alpha$ and $\xi$ are independent of the parameters $Q$ and $\beta$, which can be determined from measurements of the differential cross section. We have demonstrated in this paper that the combination of the experimental results for these five parameters can be used to completely decouple the photoionization channels, in the sense that the transition matrix elements and their relative phases can be determined for every single dissociation channel separately.

This was performed for the photoionization of xenon atoms in the $5 p$-shell in the wavelength range from the autoionization region at about $100 \mathrm{~nm}$ up to $40 \mathrm{~nm}$ in the continuous spectral range. The experimental results are well reproduced by ab initio calculations in RPAE and RRPA, thus demonstrating the high standard and the reliability of the sophisticated photoionization theories for closedshell systems. The Multichannel Quantum-Defect Theory (MQDT) was used to compare the basic photoionization quantities, transition matrix elements and their relative phases, in the discrete and in the continuous spectral range. Some of these quantities show energy dependences in the continuum, which cannot be inferred from their behaviour in the discrete region. This is due to the strong influence of the spin-orbit interaction on the continuum states and to correlation effects, which most clearly show up when the dissociation channels are classified according to the angular-momentumtransfer formalism.

Some specific experimental difficulties arising in spin- and angle-resolved photoelectron spectroscopy of gaseous targets with circularly polarized radiation were mentioned in the experimental section. When count rates are below 1 count/s in the Mott detector, accumulation times of more than a day may be necessary to measure a single spin-polarization value at a certain reaction angle. Access to a light source with good long-time stability and high flux of circularly polarized photons is thus an essential requirement for such experiments. In the near future, the rapid progress in machine techniques will lead to the construction of pure wiggler- and undulator machines, which may yield photon fluxes two orders of magnitude higher than do the present storage rings. Intensity problems in gas-phase experiments may thereby be drastically reduced. In addition, the de- 
velopment of helical wigglers may open up the possibility to use circularly polarized radiation not only in the normal-incidence range, but also at photon energies above $40 \mathrm{eV}$ [135]. The method of spinand angle-resolved photoelectron spectroscopy can thus be extended to inner-shell photoionization.

Although synchrotron radiation is an attractive tool for such experiments, the limited resolution associated with its monochromatization is sometimes not sufficient for the detailed investigation of resonance phenomena and of the photoionization of molecules. Circularly polarized radiation with extremely small bandwidth in the vacuum ultraviolet may for such cases be provided by frequency-doubling, tripling and mixing of light from high-power dye-laser systems [136].

Apart from these possible technical developments, we see a number of applications of spin- and angle-resolved photoelectron spectroscopy with circularly polarized radiation on the basis of the present possibilities. In particular, the potential of the apparatus described in this paper is by no means exhausted. Recently, we have finished a series of measurements on the photoionization of mercury, where autoionization and ionization in the $5 d$-shell in the continuum region were investigated. The excellent running conditions of the storage ring BESSY (up to $800 \mathrm{~mA}$ beam current) even allowed measurements near the Cooper-minimum of $\mathrm{Hg} 6 \mathrm{~s}$, where the cross section is below $1 \mathrm{Mb}$. In the last weeks, a high-temperature atomic beam oven went into operation, and first spin-polarization measurements on indium atoms were performed. With this atomic beam source, open-shell systems can be investigated, where theoretical methods beyond the RRPA are expected to be necessary, as is already the case in $\mathrm{Hg} 6 \mathrm{~s}$-ionization $[17,22]$.

The application of the method is, of course, not restricted to photoionization in the gas phase. In fact, the apparatus described in this paper shares the beamline at BESSY with a similar experiment, where spin-polarized photoemission from solids and adsorbates is investigated [137]. Especially in photoemission from adsorbates, surprising similarities arise with the photoionization in the gas phase. Narrow $\mathrm{Xe} 5 p \rightarrow 6 s$ resonances $(\Delta E<80 \mathrm{meV})$ have been observed to persist in the adsorbate phase [138]. The photon-induced transitions reveal an additional level splitting compared to the gas phase, which is a consequence of symmetry lowering on the surface. Furthermore, pronounced autoionization-like features with strongly enhanced intensities occur just above the $\mathrm{Xe} 5 p$ adsorbate photoemission threshold [139].

Since experiments employing spin- and angle-re- solved photoelectron spectroscopy are already routinely running for several years, we believe that this method has evolved from a rather exotic status to a powerful tool. For a number of different systems, the method has proven to provide detailed information on photoemission dynamics, which cannot be obtained by any other technique. Such experiments are thus complementary to and not competing with intensity spectroscopy. In view of the forthcoming development of a new generation of synchrotron radiation and VUV laser sources, the conditions for the full exploitation of the information contained in the spin orientation of photoelectrons will further improve in the near future.

It is a pleasure to thank the staff of BESSY for friendly cooperation in an encouraging atmosphere. We acknowledge support by the Bundesministerium für Forschung und Technologie.

\section{References}

1. Starace, A.F.: Corpuscles and Radiation in Matter 1. In: Handbuch der Physik. Mehlhorn, W. (Ed), Vol. 31, p. 1. Berlin, Heidelberg, New York: Springer 1982

2. Berkowitz, J.: Photoabsorption, Photoionization and Photoelectron Spectroscopy. p. 155 ff. New York, San Francisco, London: Academic Press 1979

3. Kessler, J.: Polarized electrons. In: Texts and Monographs in Physics. p. 14 ff. Berlin: Springer 1976 (2nd Edn. 1985)

4. Fano, U.: Phys. Rev. 178, 131 (1969)

5. Fano, U.: Phys. Rev. 184, 250 (1969)

6. Heinzmann, U., Kessler, J., Lorenz, J.: Phys. Rev. Lett. 25, $1325(1970)$

7. Heinzmann, U., Kessler, J., Lorenz, J.: Z. Phys. 240, 42 (1970)

8. Kessler, J.: In: Physics of electronic and atomic collisions. Datz, S. (ed.), p. 467. Amsterdam, New York, Oxford: North Holland 1982

9. Heinzmann, U.: Appl. Opt. 19, 4087 (1980)

10. Heinzmann, U., Schönhense, G., Wolcke, A.: In: Coherence and correlations in atomic collisions. Kleinpoppen, $\mathrm{H}$., Williams, J.F. (eds.), p. 607. New York, London: Plenum Press 1980

11. Heinzmann, U., Heuer, H., Kessler, J.: Phys. Rev. Lett. 34, $441(1975)$

12. Heinzmann, U., Heuer, H., Kessler, J.: Phys. Rev. Lett. 36, 1444 (1976)

13. Heinzmann, U.: J. Phys. B 11, 399 (1978)

14. Heinzmann, U., Wolcke, A., Kessler, J.: J. Phys. B 13, 3149 (1980)

15. Heinzmann, U., Schäfers, F., Thimm, K., Wolcke, A., Kessler, J.: J. Phys. B 12, L679 (1979)

16. Heinzmann, U., Schäfers, F.: J. Phys. B 13, L415 (1980)

17. Schäfers, F., Schönhense, G., Heinzmann, U.: Z. Phys. A Atoms and Nuclei 304, 41 (1982)

18. Schönhense, G., Schäfers, F., Heinzmann, U., Kessler, J.: Z. Phys. A - Atoms and Nuclei 304, 31 (1982)

19. Heckenkamp, Ch., Schäfers, F., Heinzmann, U., Frey, R., Schlag, E.W.: Nucl. Instrum. Methods 208, 805 (1983)

20. Heinzmann, U., Schäfers, F., Hess, B.A.: Chem. Phys. Lett. 69, 284 (1980) 
21. Heinzmann, U., Osterheld, B., Schäfers, F., Schönhense, G.: J. Phys. B 14, L79 (1981)

22. Schönhense, G., Schäfers, F., Heckenkamp, Ch., Heinzmann, U., Baig, M.A.: J. Phys. B 17, L 771 (1984)

23. Heinzmann, U,, Schönhense, G., Kessler, J.: Phys. Rev. Lett. 42, 1603 (1979)

24. Heinzmann, U., Schönhense, G., Kessler, J.: J. Phys. B13, L153 (1980)

25. Schönhense, G.: Phys. Rev. Lett. 44, 640 (1980)

26. Heinzmann, U.: J. Phys. B 13, 4353 (1980)

27. Schönhense, G., Heinzmann, U., Kessler, J., Cherepkov, N.A.: Phys. Rev. Lett. 48, 603 (1982)

28. Stewart, H.A.: Phys. Rev. A2, 2260 (1970)

29. Brehm, B.: Z. Phys. 242, 195 (1971)

30. Jacobs, V.L.: J. Phys. B 5, 2257 (1972)

31. Cherepkov, N.A.: Phys. Lett. 40 A, 119 (1972)

32. Cherepkov, N.A.: Zh. Eksp. Teor. Fiz. 65, 933 (1973); (Sov. Phys. JETP 38, 463 (1974))

33. Lee, C.M.: Phys. Rev. A 10, 1598 (1974)

34. Klar, H.: J. Phys. B 13, 3117 (1980)

35. Huang, K.N.: Phys. Lett. A77, 133 (1980)

36. Huang, K.N.: Phys. Rev. A 22, 223 (1980)

37. Cherepkov, N.A.: Adv. At. Mol. Phys. 19, 395 (1983)

38. Cherepkov, N.A.: J. Phys. B 11, L435 (1978)

39. Cherepkov, N.A.: J. Phys. B12, 1279 (1979)

40. Cherepkov, N.A.: Phys. Lett. 66 A, 204 (1978)

41. Huang, K.N., Johnson, W.R., Cheng, K.T.: At. Data Nucl. Data Tables 26, 33 (1981)

42. Cheng, K.T., Huang, K.N., Johnson, W.R.: J. Phys. B 13, L 45 (1980)

43. Huang, K.N., Starace, A.F.: Phys. Rev. A21, 697 (1980)

44. Johnson, W.R., Radojevic, V., Deshmukh, V.P., Cheng, K.T. Phys. Rev. A25, 337 (1982)

45. Huang, K.N., Starace, A.F.: Phys. Rev. A 19, 2335 (1979)

46. Huang, K.N., Starace, A.F.: Phys. Rev. A 22, 318 (1980)

47. Parpia, F.A., Johnson, W.R., Radojevic, V.: Phys. Rev. A 29, 3173 (1984)

48. Cherepkov, N.A.: J. Phys. B 10, L653 (1977)

49. Cherepkov, N.A.: Opt. Spectrosc. 49, $1067(1980)$

50. Radojevic, V., Johnson, W.R.: J. Phys. B16, 177 (1983)

51. Kabachnik, N.M., Sazhina, L.P.: J. Phys. B9, 1681 (1976)

52. Johnson, W.R., Cheng, K.T., Huang, K.N., Le Dourneuf, M.: Phys. Rev. A 22, 989 (1980)

53. Cherepkov, N.A.: J. Phys. B13, L181 (1980)

54. Schäfers, F., Schönhense, G., Heinzmann, U.: Phys. Rev. A 28, 802 (1983)

55. Heckenkamp, Ch., Schäfers, F., Schönhense, G., Heinzmann, U.: Phys. Rev. Lett. 52, 421 (1984)

56. Heckenkamp, Ch., Schäfers, F., Schönhense, G., Heinzmann, U.: Phys. Rev. A 32, 1252 (1985)

57. Heinzmann, U.: J. Phys. B 13, 4367 (1980)

58. Kessler, J.: Comm. At. Mol. Phys. 10, 47 (1981)

59. Amusia, M.Ya.: Comm. At. Mol. Phys. 8, 61 (1979)

60. Amusia, M.Ya., Cherepkov, N.A.: Case Stud. At Phys. 5, 47 (1975)

61. Yang, C.N,: Phys. Rev. 74, 764 (1948)

62. Peshkin, M.: Adv. Chem. Phys, 18, 1 (1970)

63. Samson, J.A.R.: J. Opt. Soc. Am. 59, 356 (1969)

64. Bartschat, K., Scott, P.: J. Phys. B 18, 3725 (1985)

65. Schmidt, V.: Phys. Lett. 45 A, 63 (1973)

66. Samson, J.A.R., Starace, A.F.: J. Phys. B 8, 1806 (1975)

67. Samson, J.A.R., Starace, A.F.; J. Phys. B 12, 3993 (1979)

68. Jost, K.: J. Phys. E 12, 1001 and 1006 (1979)

69. Heckenkamp, Ch., Eyers, A., Schäfers, F., Schönhense, G., Heinzmann, U.: Nucl. Instrum. Methods (in press)

70. Eyers, A., Heckenkamp, Ch., Schäfers, F., Schönhense, G., Heinzmann, U.: Nucl. Instrum. Methods 208, 303 (1983)
71. Schäfers, F., Peatman, W., Eyers, A., Heckenkamp, Ch., Schönhense, G., Heinzmann, U.: Rev. Sci. Instrum. 57, 1032 (1986)

72. Heinzmann, U.: Habilitationsschrift, University of Münster (1979), unpublished and private communication

73. Samson, J.A.R.: J. Opt. Soc. Am. 54, 842 (1964)

74. Weissler, G.L.: J. Quant. Spectrosc. Radiat. Transfer 2, 383 (1962)

75. Samson, J.A.R.: Adv. At. Mol. Phys. 2, 177 (1966)

76. Hudson, R.D., Kieffer, L.J.: At. Data 2, 205 (1970)

77. Metzger, D.H., Cook, G.R.: J. Opt. Soc. Am. 55, $516(1965)$

78. Matsunaga, F.M., Jackson, R.S., Watanabe, K.: J. Quant. Spectrosc. Radiat. Transfer 5, 329 (1965)

79. Carter, V.L., Hudson, R.D.: J. Opt. Soc. Am. 63, 733 (1973)

80. Blackwell, H.E., Bagwa, G.S., Shipp, G.S., Weissler, G.L.: J. Quant. Spectrosc. Radiat. Transfer 4, 249 (1964)

81. Rustgi, O.P., Fisher, E.J., Fuller, C.H.: J. Opt. Soc. Am. 54, 745 (1964)

82. Huffman, R.E., Tanaka, Y., Larrabee, J.C.: J. Chem. Phys. 39, 902 (1963)

83. West, J.B., Morton, J.: At. Data Nucl. Tables 22, 103 (1978)

84. Wuilleumier, F., Adam, M.Y., Dhez, P., Sandner, N., Schmidt, V., Mehlhorn, W.: Phys. Rev. A 16, 646 (1977)

85. Adam, M.Y., Wuilleumier, F., Krummacher, S., Sandner, N., Schmidt, V., Mehlhorn, W.: J. Electron Spectrose. 15, 211 (1979)

86. Levinson, H.J., McGovern, D.T., Gustafsson, T.: J. Phys. B 13, 253 (1980)

87. Codling, K., West, J.B., Parr, A.C., Dehmer, J.L., Stockbauer, R.L.: J. Phys. B 13, L693 (1980)

88. Ederer, D.L., Parr, A.C., West, J.B., Holland, D., Dehmer, J.L.: Phys. Rev. A25, 2006 (1982)

89. Krause, M.O., Carlson, T.A., Woodruff, P.R.: Phys. Rev. A24, 1374 (1981)

90. Frost, D.C., McDowell, C.A., Vroom, D.A.: Proc. R. Soc. London Ser. A 296, 566 (1976)

91. Turner, D.W., May, D.P.: J. Chem. Phys. 45, 471 (1966)

92. Rabalais, J.W., Debies, T.B.: J. Electron. Spectrosc. 5, 847 (1974)

93. Comes, F.J., Sälzer, H.G.: Z. Naturforsch. 119, 1230 (1964)

94. Samson, J.A.R., Gardner, J.L.: J. Opt. Soc. Am. 62, 856 (1972)

95. Samson, J.A.R., Cairns, R.B.: Phys. Rev. 173, 80 (1968)

96. Samson, J.A.R., Gardner, J.L., Starace, A.F.: Phys. Rev. A 12, $1459(1975)$

97. Samson, J.A.R.: J. Electron. Spectrosc. 15, 257 (1979)

98. Kibel, M.H., Leng, F.J., Nyberg, G.L.: J. Electron. Spectrosc. 15, 281 (1979)

99. Berkowitz, J., Ehrhardt, H.: Phys. Lett. 21, 531 (1966)

100. McGowan, J.W., Vroom, D.A., Comeaux, A.R.: J. Chem. Phys. 51, 5626 (1969)

101. Samson, J.A.R.: J. Opt. Soc. Am. 59, 356 (1969)

102. Carlson, T.A., Jonas, A.E.: J. Chem. Phys. 55, 4913 (1971)

103. Niehaus, A., Ruf, M.W.: Z. Phys. 252, 84 (1972)

104. Dehmer, J.L., Chupka, W.A., Berkowitz, J., Jivery, W.T.: Phys. Rev. A 12, 1966 (1975)

105. Hancock, W.H., Samson, J.A.R.: J. Electron. Spectrosc. 9, 211 (1976)

106. Kreile, J., Schweig, A.: J. Electron. Spectrosc. 20, 191 (1980)

107. Schönhense, G.: Diploma Thesis, University of Münster 1978 (unpublished)

108. Holland, D.M.P., Parr, A.C., Ederer, D.L., Dehmer, J.L., West, J.B.: Nucl. Instrum. Methods 195, 331 (1982)

109. Lynch, M.J, Codling, K., Gardner, A.B.: Phys. Lett. 43 A, $213(1973)$

110. Seaton, M.J.: Comm. At. Mol. Phys. 2, 37 (1970)

111. Fano, U.: J. Opt. Soc. Am. 65, 979 (1975) 
112. Starace, A.F.: In: Photoionization and other probes of many electron interactions. Wuilleumier, F. (ed.), p. 395. New York: Plenum Press 1976

113. Geiger, J.: Z. Phys. A - Atoms and Nuclei 282, 129 (1977)

114. Geiger, J.: Z. Phys. A - Atoms and Nuclei 276, 219 (1976)

115. Dill, D.: Phys. Rev. A7, 1976 (1973)

116. Manson, S.T.: Adv, Electron. Electron Phys. 41, 43 (1976) and 44, 1 (1977)

117. Burgess, A.: Proc. Phys. Soc, 81, 442 (1963)

118. Geiger, J.: Private communication (1979)

119. Amusia, M.Ya., Ivanov, V.K.: Izv. Akad. Nauk SSSR, Ser. Fiz. 41, 2509 (1977), see also Ref, 37

120. Dill, D., Fano, U.: Phys. Rev. Lett. 29, 1203 (1972)

121. Fano, U., Dill, D.: Phys. Rev. A6, 185 (1972)

122. Dill, D.: In: Photoionization and other probes of many electron interactions. Wuilleumier, F. (ed.), p. 387. New York: Plenum Press 1976

123. Dill, D., Starace, A.F., Manson, S.T.: Phys. Rev. A 11, 1569 (1975)

124. Dill, D., Dehmer, J.L.: J. Chem. Phys. 61, 692 (1974)

125. Dehmer, J.L., Dill, D.: Phys. Rev. Lett. 35, 213 (1975)

126. Beutler, H.: Z. Phys. 93, 177 (1935)

127. Fano, U.: Nuovo Cimento 12, $156(1935)$

128. Fano, U.: Phys. Rev. 124, 1866 (1961)

129. Saile, V.: PhD-Thesis, University of München (1976), unpublished

130. Eland, J.H.D.: unpublished results, see Ref. 2, p. 181

131. Samson, J.A.R., Gardner, J.L.: Phys. Rev. Lett. 22, 1327 (1973); Private communication (1984)

132. Morioka, Y., Watanabe, M., Akahori, T., Yagishita, A., Nakamura, M.: J. Phys. B18, 71 (1985)
133. Lee, C.M., Johnson, W.R.: Phys. Rev. A22, 979 (1980)

134. Lu, K.T.: Phys. Rev. A 4, 579 (1971)

135. Kim, K.I.: In: Applications of circularly polarized radiation using synchrotron and ordinary sources. Allen, $F_{y}$, Bustamante, C. (eds.), p. 21. New York: Plenum Press 1985; Nucl. Instrum. Methods 219, 425 (1984)

136. Wallenstein, R.: Laser Optoelektron. 14, 29 (1982)

137. Heinzmann, U., Schönhense, G.: In: Polarized electrons in surface physics. Feder, R. (ed.), Chap. 11, p. 467. Singapore: World Scientific 1985

138. Schönhense, G., Eyers, A., Heinzmann, U.: Phys. Rev. Lett. 56, 512 (1986)

139. Schönhense, G., Eyers, A., Friess, U., Schäfers, F., Heinzmann, U.: Phys. Rev. Lett. 54, 547 (1985)

Ch. Heckenkamp

F. Schäfers

G. Schönhense

U. Heinzmamn

Fakultät für Physik

Universität Bielefeld

D.4800 Bielefeld

Federal Republic of Germany

and

Fritz-Haber-Institut der MPG

Faradayweg 4-6

D-1000 Berlin 33

Federal Republic of Germany 